Article

\title{
Synthesis and Bioactivity of Thiosemicarbazones Containing Adamantane Skeletons
}

\author{
Van Hien Pham ${ }^{1}$, Thi Phuong Dung Phan ${ }^{2}$, Dinh Chau Phan ${ }^{3, *}$ and Binh Duong Vu ${ }^{1, *}$ \\ 1 Drug R\&D Center, Vietnam Military Medical University. No.160, Phung Hung Street., Phuc La ward, \\ Ha Dong District, Hanoi 100000, Vietnam; phamvanhien181288@gmail.com \\ 2 Department of Pharmaceutical Chemistry, Hanoi University of Pharmacy. No. 15, Le Thanh Tong Street, \\ Hoan Kiem District, Hanoi 100000, Vietnam; pdungdhd@gmail.com \\ 3 Hanoi University of Science and Technology. No.1, Dai Co Viet Street., Bach Khoa Ward, Hai Ba Trung \\ District, Hanoi 100000, Vietnam \\ * Correspondence: chau.phandinh@hust.edu.vn (D.C.P.); vbduong2978@gmail.com (B.D.V.); \\ Tel.: +84 983425460 (B.D.V.); Fax: +84 2436884077 (B.D.V.)
}

Received: 17 December 2019; Accepted: 9 January 2020; Published: 13 January 2020

\begin{abstract}
Reaction of 4-(1-adamantyl)-3-thiosemicarbazide (1) with numerous substituted acetophenones and benzaldehydes yielded the corresponding thiosemicarbazones containing adamantane skeletons. The synthesized compounds were evaluated for their in vitro activities against some Gram-positive and Gram-negative bacteria, and the fungus Candida albicans, and cytotoxicity against four cancer cell lines (Hep3B, HeLa, A549, and MCF-7). All of them showed good antifungal activity against Candida albicans. Compounds $\mathbf{2 c}, \mathbf{2 d}, \mathbf{2 g}, \mathbf{2 j}$ and $\mathbf{3 a}, \mathbf{3 e}, \mathbf{3 g}$ displayed significant inhibitory activity against Enterococcus faecalis. Compounds $\mathbf{2 a}, \mathbf{2 e}, \mathbf{2} \mathbf{h}, \mathbf{2 k}$ and $\mathbf{3} \mathbf{j}$ had moderate inhibitory potency against Staphylococcus aureus. Compounds $2 \mathbf{a}, \mathbf{2 e}$ and $\mathbf{2 g}$ found so good inhibitory effect on Bacillus cereus. Compounds $\mathbf{2} \mathbf{d}$ and $\mathbf{2 h}$, which contain (ortho) hydroxyl groups on the phenyl ring, were shown to be good candidates as potential agents for killing the tested cancer cell lines, i.e., Hep3B, A549, and MCF-7. Compounds $\mathbf{2 a - c}, \mathbf{2 f}, \mathbf{2} \mathbf{g}, \mathbf{2 j}, \mathbf{2 k}, \mathbf{3 g}$, and $\mathbf{3 i}$ were moderate inhibitors against MCF-7.
\end{abstract}

Keywords: adamantane derivatives; thiosemicarbazone; antimicrobial; cytotoxicity activity

\section{Introduction}

Thiosemicarbazone derivatives, which play an important role in organic and medicinal chemistry, have attracted a large number of researchers over the years because of their promising biological activities, such as antioxidant [1,2], antiparasitic [3-6], anticonvulsant [7], antiviral [8-10]. Especially, in recent years, large of publications focused on synthesis and antimicrobial [11-15], anticancer [12,14, 16-20] activity of thiosemicarbazones to find out the potential candidates to develop new drugs.

Since the first adamantane derivative, amantadine, was found to have antiviral [21-23] activity, the synthesis and biological activities of adamantane derivatives, which became an interesting topic, were pursued by a lot of researchers. Therefore, many adamantane derivatives were discovered with various biological activities, i.e., antiviral [24-29], antimicrobial [27,29-39], anticancer [32,36,40-44] activities.

To continue to find out the modern molecules, which possess potential bioactivities and contain adamantane skeleton following our previous research [45], herein, we combined two moieties, thiosemicarbazone constituent and adamantane skeleton, which potentially help to confer new molecules with promising biological activities. In this publication, we report the synthesis and biological activities of thiosemicarbazones containing adamantane skeleton. 


\section{Results and Discussion}

\subsection{Chemistry}

In this study, 4-(1-adamantyl)-3-thiosemicarbazide (1) was used as the key initial material. The condensation of $\mathbf{1}$ and substituted benzaldehydes under the catalysis of acetic acid and reflux condition in $\mathrm{MeOH}$ to yield thiosemicarbazones 2a-k (Scheme 1). Similarly, compound $\mathbf{1}$ was condensed with substituted acetophenones to yield thiosemicarbazones $\mathbf{3 a} \mathbf{a} \mathbf{j}$ as showed in Scheme 1 and Table 1 . The structure of synthesized $\mathbf{2} \mathbf{a}-\mathbf{k}$ and $\mathbf{3 a} \mathbf{a}-\mathbf{j}$ was confirmed by nuclear magnetic resonance, including ${ }^{1} \mathrm{H}-\mathrm{NMR},{ }^{13} \mathrm{C}-\mathrm{NMR}$, and electron impact (ESI-MS) mass spectral data.

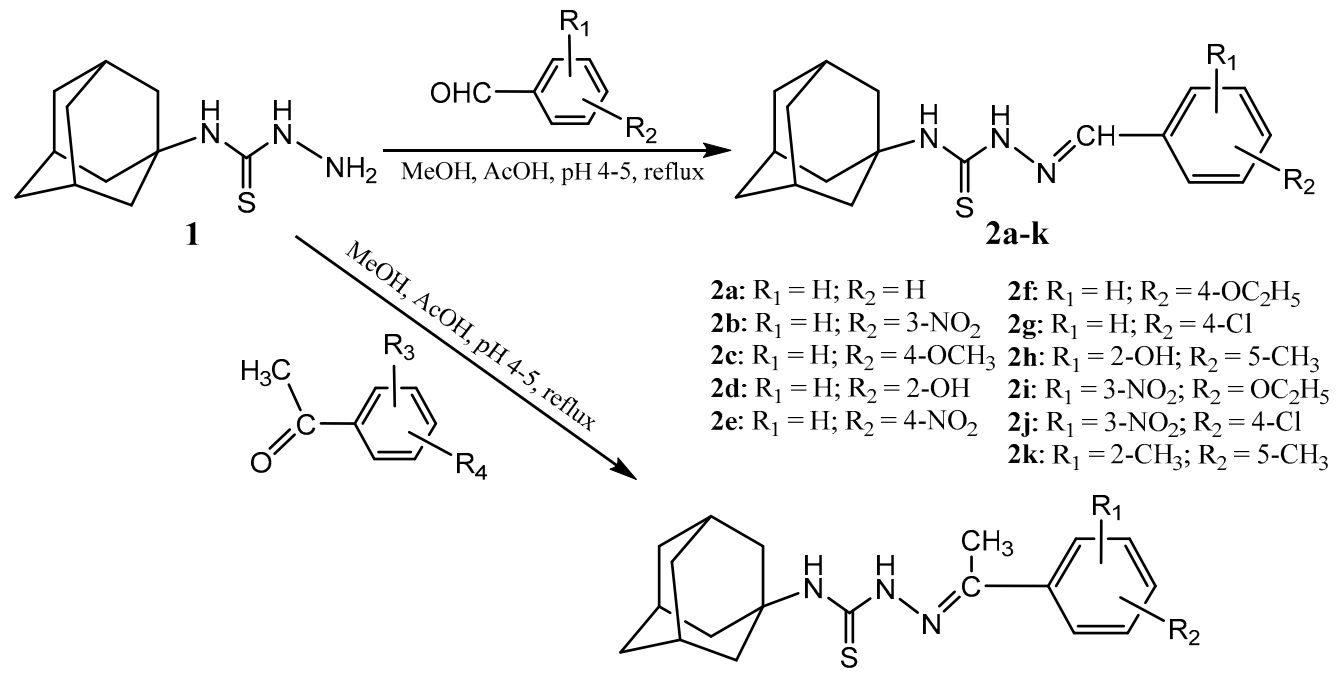

3a-k

$$
\begin{array}{ll}
\text { 3a: } \mathrm{R}_{1}=H ; \mathrm{R}_{2}=\mathrm{H} & \text { 3f: } \mathrm{R}_{1}=3-\mathrm{NO}_{2} ; \mathrm{R}_{2}=4-\mathrm{Br} \\
\text { 3b: } \mathrm{R}_{1}=H ; \mathrm{R}_{2}=3-\mathrm{NO}_{2} & \text { 3g: } \mathrm{R}_{1}=\mathrm{H} ; \mathrm{R}_{2}=4-\mathrm{Cl} \\
\text { 3c: } \mathrm{R}_{1}=H ; \mathrm{R}_{2}=4-\mathrm{Br} & \text { 3h: } \mathrm{R}_{1}=\mathrm{H} ; \mathrm{R}_{2}=4-\mathrm{CH}_{3} \\
\text { 3d: } \mathrm{R}_{1}=H ; \mathrm{R}_{2}=4-\mathrm{OH} & \text { 3i: } \mathrm{R}_{1}=3-\mathrm{NO}_{2} ; \mathrm{R}_{2}=4-\mathrm{OCH}_{3} \\
\text { 3e: } \mathrm{R}_{1}=\mathrm{H} ; \mathrm{R}_{2}=4-\mathrm{NO}_{2} & \text { 3j: } \mathrm{R}_{1}=3-\mathrm{NO}_{2} ; \mathrm{R}_{2}=4-\mathrm{Cl}
\end{array}
$$

\begin{tabular}{|c|c|c|c|c|c|c|}
\hline Component & $\mathbf{R} 1$ & $\mathbf{R} 2$ & $\begin{array}{l}\text { Yield } \\
(\%)\end{array}$ & m.p $\left({ }^{\circ} \mathrm{C}\right)$ & $\begin{array}{l}\text { Molecular Formulae } \\
\text { (Molecular Weight) }\end{array}$ & $\mathbf{R f}$ \\
\hline $2 a$ & $\mathrm{H}$ & $\mathrm{H}$ & 97.0 & $210.1-212.2$ & $\mathrm{C}_{18} \mathrm{H}_{23} \mathrm{~N}_{3} \mathrm{~S}$ (313.46) & 0.46 \\
\hline $2 b$ & $\mathrm{H}$ & $3-\mathrm{NO}_{2}$ & 92.7 & $244.2-246.1$ & $\mathrm{C}_{18} \mathrm{H}_{22} \mathrm{~N}_{4} \mathrm{O}_{2} \mathrm{~S}$ (358.46) & 0.38 \\
\hline $2 c$ & $\mathrm{H}$ & $4-\mathrm{OCH}_{3}$ & 95.7 & $224.5-227.7$ & $\mathrm{C}_{19} \mathrm{H}_{25} \mathrm{~N}_{3} \mathrm{OS}$ (343.49) & 0.50 \\
\hline $2 \mathrm{~d}$ & $\mathrm{H}$ & $2-\mathrm{OH}$ & 95.7 & 203.8-205.6 & $\mathrm{C}_{18} \mathrm{H}_{23} \mathrm{~N}_{3} \mathrm{OS}(329.46)$ & 0.46 \\
\hline $2 e$ & $\mathrm{H}$ & $4-\mathrm{NO}_{2}$ & 91.3 & $258.1-260.1$ & $\mathrm{C}_{18} \mathrm{H}_{22} \mathrm{~N}_{4} \mathrm{O}_{2} \mathrm{~S}$ (358.46) & 0.43 \\
\hline $2 f$ & $\mathrm{H}$ & $4-\mathrm{OC}_{2} \mathrm{H}_{5}$ & 95.6 & $232.2-233.6$ & $\mathrm{C}_{20} \mathrm{H}_{27} \mathrm{~N}_{3} \mathrm{OS}(357.52)$ & 0.54 \\
\hline $2 \mathrm{~g}$ & $\mathrm{H}$ & 4-Cl & 89.6 & $238.9-239.7$ & $\mathrm{C}_{18} \mathrm{H}_{22} \mathrm{ClN}_{3} \mathrm{~S}$ (347.91) & 0.68 \\
\hline $2 \mathrm{~h}$ & $2-\mathrm{OH}$ & $5-\mathrm{CH}_{3}$ & 91.0 & $241.6-242.5$ & $\mathrm{C}_{19} \mathrm{H}_{25} \mathrm{~N}_{3} \mathrm{OS}$ (343.49) & 0.54 \\
\hline $2 \mathbf{i}$ & $3-\mathrm{NO}_{2}$ & $4-\mathrm{OC}_{2} \mathrm{H}_{5}$ & 61.2 & $218.7-220.7$ & $\mathrm{C}_{20} \mathrm{H}_{26} \mathrm{~N}_{4} \mathrm{O}_{3} \mathrm{~S}(402.51)$ & 0.64 \\
\hline $2 j$ & $3-\mathrm{NO}_{2}$ & 4-Cl & 78.5 & $252.8-254.0$ & $\mathrm{C}_{18} \mathrm{H}_{21} \mathrm{ClN}_{4} \mathrm{O}_{2} \mathrm{~S}$ (392.90) & 0.53 \\
\hline $2 k$ & $2-\mathrm{CH}_{3}$ & $5-\mathrm{CH}_{3}$ & 92.5 & $212.4-213.8$ & $\mathrm{C}_{20} \mathrm{H}_{27} \mathrm{~N}_{3} \mathrm{~S}(341.52)$ & 0.68 \\
\hline $3 \mathbf{a}$ & $\mathrm{H}$ & $\mathrm{H}$ & 91.8 & $231.2-232.7$ & $\mathrm{C}_{19} \mathrm{H}_{25} \mathrm{~N}_{3} \mathrm{~S}$ (327.49) & 0.46 \\
\hline $3 b$ & $\mathrm{H}$ & $3-\mathrm{NO}_{2}$ & 67.0 & $251.7-253.5$ & $\mathrm{C}_{19} \mathrm{H}_{24} \mathrm{~N}_{3} \mathrm{O}_{2} \mathrm{~S}$ (372.49) & 0.47 \\
\hline $3 c$ & $\mathrm{H}$ & $4-\mathrm{Br}$ & 65.5 & $240.9-242.9$ & $\mathrm{C}_{19} \mathrm{H}_{24} \mathrm{BrN}_{3} \mathrm{~S}$ (406.39) & 0.46 \\
\hline $3 d$ & $\mathrm{H}$ & $4-\mathrm{OH}$ & 44.3 & $272.8-273.5$ & $\mathrm{C}_{19} \mathrm{H}_{25} \mathrm{~N}_{3} \mathrm{OS}$ (343.49) & 0.53 \\
\hline $3 e$ & $\mathrm{H}$ & $4-\mathrm{NO}_{2}$ & 90.4 & $266.5-268.9$ & $\mathrm{C}_{19} \mathrm{H}_{24} \mathrm{~N}_{4} \mathrm{O}_{2} \mathrm{~S}$ (372.49) & 0.50 \\
\hline $3 f$ & $3-\mathrm{NO}_{2}$ & $4-\mathrm{Br}$ & 17.5 & $224.5-225.3$ & $\mathrm{C}_{19} \mathrm{H}_{23} \mathrm{BrN}_{4} \mathrm{O}_{2} \mathrm{~S}$ (451.38) & 0.53 \\
\hline $3 g$ & $\mathrm{H}$ & $4-\mathrm{Cl}$ & 94.0 & $235.0-236.3$ & $\mathrm{C}_{19} \mathrm{H}_{24} \mathrm{ClN}_{3} \mathrm{~S}$ (361.93) & 0.58 \\
\hline $3 \mathrm{~h}$ & $\mathrm{H}$ & $4-\mathrm{CH}_{3}$ & 73.5 & $230.3-232.2$ & $\mathrm{C}_{20} \mathrm{H}_{27} \mathrm{~N}_{3} \mathrm{~S}(341.52)$ & 0.36 \\
\hline $3 \mathbf{i}$ & $3-\mathrm{NO}_{2}$ & $4-\mathrm{OCH}_{3}$ & 69.3 & $224.6-226.3$ & $\mathrm{C}_{20} \mathrm{H}_{26} \mathrm{~N}_{4} \mathrm{O}_{3} \mathrm{~S}(402.51)$ & 0.45 \\
\hline $3 \mathbf{j}$ & $3-\mathrm{NO}_{2}$ & 4-Cl & 49.8 & 250.9-252.4 & $\mathrm{C}_{19} \mathrm{H}_{23} \mathrm{ClN}_{4} \mathrm{O}_{2} \mathrm{~S}(406.93)$ & 0.54 \\
\hline
\end{tabular}

Scheme 1. Synthesis of thiosemicarbazones $\mathbf{2 a - k}$ and $3 \mathbf{a}-\mathbf{j}$.

Table 1. Melting point (m.p), yield (\%), molecular formulae (Mol.For.), molecular weight (Mol. Wt.) and Rf of thiosemicarbazones $\mathbf{2} \mathbf{a}-\mathbf{k}$ and $\mathbf{3 a}-\mathbf{j}$.

Solvent: chloroform/acetone $(95 / 5, v / v)$, visualization at UV $254 \mathrm{~nm}$. Rf: retention factor. 


\subsection{In Vitro Antimicrobial Activity}

The synthesized thiosemicarbazones $\mathbf{2} \mathbf{a}-\mathbf{k}$ and $\mathbf{3 a}-\mathbf{j}$ were evaluated for their in vitro growing inhibition against seven strains of the National Institute for Food Control (NIFC, Hanoi, Vietnam), including three Gram-negative bacteria (Escherichia coli ATCC25922, Pseudomonas aeruginosa ATCC27853, and Salmonella enterica ATCC12228); three Gram-positive bacteria (Enterococcus faecalis ATCC13124, Staphylococcus aureus ATCC25923, and Bacillus cereus ATCC 13245); and one yeast-like pathogenic fungus, Candida albicans ATCC10231. The primary screening was conducted using the microplate dilution method, which utilized Luria-Bertani (LB) broth medium. Streptomycin (an antibiotic) and cycloheximide (an antifungal agent) were used as the positive samples. The results of preliminary antimicrobial testing of the synthesized thiosemicarbazones $\mathbf{2} \mathbf{a}-\mathbf{k}$ and $\mathbf{3} \mathbf{a}-\mathbf{j}$ are presented in Tables 2 and 3 .

Table 2. Minimum inhibitory concentration (MIC) of synthesized thiosemicarbazones $\mathbf{2 a}-\mathbf{k}$ and $\mathbf{3 a} \mathbf{a} \mathbf{j}$.

\begin{tabular}{|c|c|c|c|c|c|c|c|}
\hline \multirow{3}{*}{ Comp. No. } & \multicolumn{7}{|c|}{ MIC of Synthesized Compounds $(\mu \mathrm{M})$} \\
\hline & \multicolumn{3}{|c|}{ Gram (+) } & \multicolumn{3}{|c|}{ Gram (-) } & \multirow{2}{*}{$\frac{\text { Fungus }}{\text { CA }}$} \\
\hline & EF & SA & BC & EC & PA & SE & \\
\hline $2 a$ & 100 & 25 & 25 & - & - & - & 25 \\
\hline $2 b$ & 25 & 50 & 25 & - & - & - & 6.25 \\
\hline $2 c$ & 12.5 & 50 & 100 & - & - & - & 6.25 \\
\hline $2 d$ & 25 & - & 100 & - & 100 & - & 25 \\
\hline $2 e$ & 100 & 50 & 50 & - & - & - & 12.5 \\
\hline $2 f$ & 50 & 50 & 50 & - & - & - & 25 \\
\hline $2 \mathrm{~g}$ & 25 & 25 & 25 & - & - & - & 6.25 \\
\hline $2 h$ & 50 & 25 & 50 & - & 100 & - & 12.5 \\
\hline $2 \mathrm{i}$ & 50 & 50 & 25 & - & - & - & 12.5 \\
\hline $2 j$ & 50 & 50 & 50 & - & - & - & 25 \\
\hline $2 k$ & 100 & 25 & 50 & - & - & - & 12.5 \\
\hline $3 a$ & 25 & 25 & 50 & - & - & - & 12.5 \\
\hline $3 b$ & 100 & 25 & 25 & - & - & - & 25 \\
\hline $3 c$ & 100 & 100 & 100 & - & - & - & 25 \\
\hline $3 d$ & 50 & 50 & 50 & - & - & - & 25 \\
\hline $3 e$ & 25 & 25 & 25 & - & - & - & 6.25 \\
\hline $3 f$ & 25 & 50 & 50 & - & - & - & 25 \\
\hline $3 g$ & 25 & 100 & 100 & - & - & - & 25 \\
\hline $3 h$ & 50 & 25 & 50 & - & - & - & 12.5 \\
\hline $3 \mathbf{i}$ & 50 & 50 & 50 & - & - & - & 25 \\
\hline $3 \mathbf{j}$ & 100 & 25 & 50 & - & - & - & 12.5 \\
\hline STM & 350 & 350 & 175 & 44 & 350 & 175 & NT \\
\hline $\mathrm{CHM}$ & NT & NT & NT & NT & NT & NT & 114 \\
\hline
\end{tabular}

EF: Enterococcus faecalis (ATCC13124); SA: Staphylococcus aureus (ATCC25923); BC: Bacillus cereus (ATCC 13245); EC: Escherichia coli (ATCC25922); PA: Pseudomonas aeruginosa (ATCC27853); SE: Salmonella enterica (ATCC12228); CA: Candida albicans (ATCC10231); STM: Streptomycin; CHM: Cycloheximide; NT: not tested; - : inactive. 
Table 3. $\mathrm{IC}_{50}$ of synthesized thiosemicarbazones $\mathbf{2} \mathbf{a}-\mathbf{k}$ and $\mathbf{3 a} \mathbf{a} \mathbf{j}$.

\begin{tabular}{|c|c|c|c|c|c|c|c|}
\hline \multirow{3}{*}{ Comp. No. } & \multicolumn{7}{|c|}{$\mathrm{IC}_{50}$ of Synthesized Compounds $(\mu \mathrm{M})$} \\
\hline & \multicolumn{3}{|c|}{ Gram (+) } & \multicolumn{3}{|c|}{ Gram (-) } & \multirow{2}{*}{$\begin{array}{c}\text { Fungus } \\
\text { CA }\end{array}$} \\
\hline & EF & SA & BC & EC & PA & SE & \\
\hline $2 a$ & 24.78 & 4.78 & 4.12 & - & - & - & 6.78 \\
\hline $2 b$ & 10.78 & 8.99 & 12.45 & - & - & - & 3.57 \\
\hline $2 c$ & 5.68 & 9.66 & 8.24 & - & - & - & 3.45 \\
\hline $2 d$ & 4.89 & - & 25.22 & - & 24.67 & - & 5.35 \\
\hline $2 e$ & 25.89 & 6.78 & 6.09 & - & - & - & 5.56 \\
\hline $2 f$ & 12.78 & 7.88 & 7.82 & - & - & - & 6.35 \\
\hline $2 \mathrm{~g}$ & 6.78 & 7.89 & 6.88 & - & - & - & 3.24 \\
\hline $2 \mathrm{~h}$ & 11.67 & 6.24 & 7.56 & - & 27.45 & - & 4.57 \\
\hline $2 \mathrm{i}$ & 12.78 & 12.56 & 12.11 & - & - & - & 3.57 \\
\hline $2 j$ & 6.88 & 22.67 & 22.12 & - & - & - & 4.34 \\
\hline $2 \mathrm{k}$ & 47.89 & 6.45 & 8.49 & - & - & - & 5.68 \\
\hline $3 a$ & 6.34 & 6.99 & 12.33 & - & - & - & 3.67 \\
\hline $3 b$ & 25.89 & 8.99 & 9.91 & - & - & - & 7.89 \\
\hline $3 c$ & 28.99 & 50.22 & 40.45 & - & - & - & 5.67 \\
\hline $3 d$ & 17.89 & 21.45 & 25.89 & - & - & - & 6.78 \\
\hline $3 e$ & 4.67 & 9.23 & 10.11 & - & - & - & 3.22 \\
\hline $3 f$ & 13.57 & 21.44 & 11.88 & - & - & - & 3.67 \\
\hline $3 g$ & 4.78 & 35.67 & 32.11 & - & - & - & 5.34 \\
\hline $3 h$ & 12.56 & 7.88 & 9.85 & - & - & - & 6.79 \\
\hline $3 \mathbf{i}$ & 12.57 & 15.67 & 25.62 & - & - & - & 7.89 \\
\hline $3 \mathbf{j}$ & 35.46 & 6.46 & 7.49 & - & - & - & 4.67 \\
\hline
\end{tabular}

EF: Enterococcus faecalis ATCC13124; SA: Staphylococcus aureus ATCC25923; BC: Bacillus cereus ATCC 13245; EC: Escherichia coli ATCC25922; PA: Pseudomonas aeruginosa ATCC27853; SE: Salmonella enterica ATCC12228; CA: Candida albicans ATCC10231; -: inactive.

The results of antimicrobial testing showed that all synthesized thiosemicarbazones variously and seriously inhibited the tested Gram-positive bacteria and Candida albicans. Among them, compounds $\mathbf{2 c}, \mathbf{2} \mathbf{d}, \mathbf{2} \mathbf{g}, \mathbf{2} \mathbf{j}$ and $\mathbf{3 a}, \mathbf{3 e}, \mathbf{3} \mathbf{g}$ possessed good on inhibition against EF with a MIC (Minimal inhibitory concentration) of no more than $25 \mu \mathrm{M}$ and $\mathrm{IC}_{50}$ values of 5.68, 4.89, 6.78, 6.88 and $6.34,4.67$ and $4.78 \mu \mathrm{M}$, respectively. In term of the inhibition against $\mathrm{SA}$, compounds $\mathbf{2 \mathbf { a }}, \mathbf{2 \mathbf { e }}, \mathbf{2 \mathbf { h }}, \mathbf{2 \mathbf { k }}$, and $\mathbf{3} \mathbf{j}$ showed acceptable activity, with $\mathrm{IC}_{50}$ values of $4.78,6.78,6.24,6.45$ and $6.46 \mu \mathrm{M}$, respectively. Moreover, compounds $\mathbf{2 a}$, 2e, and $2 \mathrm{~g}$ showed inhibitory effects on BC with $\mathrm{IC}_{50}$ values of $4.12,6.09$ and $6.88 \mu \mathrm{M}$, individually. Remarkably, all synthesized thiosemicarbazones $\mathbf{2 a}-\mathbf{k}$ containing adamantane skeletons have significant inhibitory activity against CA. Nevertheless, only compounds $\mathbf{2 e}$ and $\mathbf{2 h}$ had been found to have mild inhibitory effect on PA, a Gram-negative bacterium.

Glancing at the structure-antimicrobial activity relationship of thiosemicarbazones $\mathbf{2 a}-\mathbf{k}$, inhibition against EF increased significantly for compounds possessing a (meta) -OH group (compounds $\mathbf{2 d}$ and $\mathbf{2 h}$ ) or (para) $-\mathrm{Cl}$ group (compounds $\mathbf{2 g}$ and $\mathbf{2} \mathbf{j}$ ) on the phenyl ring. However, the inhibitory activity against SA, BC, EC seem to decrease in the case of substituents on the phenyl ring. Otherwise, inhibition against $C A$ was improved with the presence of substituents on the phenyl ring.

In the series of thiosemicarbazones $\mathbf{3 a}-\mathbf{k}$, inhibitory activity against $\mathrm{EF}$ increased with the presence of only (para) $-\mathrm{NO}_{2}$ (compound 3e) or (para) $-\mathrm{Cl}$ (compound $3 \mathrm{~g}$ ) groups alone on the phenyl ring. In case of SAs, the inhibition was improved by having both (meta) $-\mathrm{NO}_{2}$ and (para) $-\mathrm{Cl}$ groups (compound $3 j$ ) on the phenyl ring. The inhibition against $\mathrm{CA}$ seems to increase when there is a $-\mathrm{NO}_{2}$ at position 4 on the phenyl ring (compound $\mathbf{3 e}$ ). Among all synthesized thiosemicarbazones $\mathbf{2 a}-\mathbf{k}$ and $\mathbf{3 a}-\mathbf{j}$, only compounds $\mathbf{2} \mathbf{d}$ and $\mathbf{2} \mathbf{h}$ showed the inhibition against PA in all tested Gram-negative bacteria but with limited activity. 


\subsection{In Vitro Cytotoxicity}

The synthesized thiosemicarbazones $\mathbf{2} \mathbf{a}-\mathbf{k}$ and $\mathbf{3 a}-\mathbf{j}$ were tested for their cytotoxicity in four human cancer cell lines, including Hep3B, HeLa, A549, and MCF-7, in line with a previous publication [46]. As the resulta in Table 4 show, some of the synthesized thiosemicarbazones exhibited antiproliferative activity against the tested cancer cell lines. Among them, compound $\mathbf{2 d}$ showed the killing availability against the tested cell lines with cell viabilities of $16.82 \pm 1.60 \%, 24.55 \pm 1.85,18.37 \pm 1.75$, and $20.17 \pm$ 1.52 in Hep3B, Hela, A549, and MCF-7 cell lines at a $100 \mu \mathrm{M}$ dose, respectively. Moreover, compound 2h was also seen to have good inhibitory effect on the tested cancer cell lines, with cell viabilities of $21.86 \pm 0.20 \%, 34.76 \pm 1.36 \%, 23.86 \pm 0.22 \%, 28.55 \pm 1.12 \%$ in Hep3B, Hela, A549, and MCF-7 cell lines at a $100 \mu \mathrm{M}$ dose, respectively. The ability to kill Hep3B, A549, and MCF-7 cancer cell lines of compounds $\mathbf{2 d}$ and $\mathbf{2 h}$ was stronger than that of camptothecin at the same concentration. Additionally, some synthesized compounds showed acceptable inhibition against the growth of MCF-7 such as compounds $\mathbf{2 a}-\mathbf{c}, \mathbf{2} \mathbf{f}, \mathbf{2 g}, \mathbf{2 j}, \mathbf{2 k}, \mathbf{3 g}$, and $\mathbf{3 i}$ with viability values of $49.35 \pm 0.79 \%, 43.31 \pm 2.63 \%, 49.02 \pm$ $1.18 \%, 39.35 \pm 1.67 \%, 33.40 \pm 0.86 \%, 45.33 \pm 2.40 \%, 42.09 \pm 0.40 \%, 46.67 \pm 1.49 \%$ at a concentration of $100 \mu \mathrm{M}$, respectively. Other compounds were seen to have a mild cytotoxicity effect on the tested cancer cell lines. The structure-cytotoxic activity relationship analysis revealed that compounds $\mathbf{2 d}$ and $\mathbf{2 h}$, which contain (ortho) - OH groups on the phenyl ring, have significantly improved killing availability on the tested cancer cell lines. However, an in depth study of the mechanism of the structure-cytotoxicity relationship should be undertaken.

Table 4. The effect of synthesized thiosemicarbazones $\mathbf{2 a - k}$ and $\mathbf{3 a}-\mathbf{j}$ on the viability of Hep3B, Hela, A549, and MCF-7 cells after $48 \mathrm{~h}$ of incubation.

\begin{tabular}{|c|c|c|c|c|c|}
\hline Comp. No. & Conc. & Нер3В & Hela & A549 & MCF-7 \\
\hline \multirow{2}{*}{$2 a$} & $30 \mu \mathrm{M}$ & $69.07 \pm 1.37$ & $71.58 \pm 1.49$ & $75.40 \pm 1.50$ & $58.80 \pm 1.23$ \\
\hline & $100 \mu \mathrm{M}$ & $64.47 \pm 0.86$ & $60.07 \pm 0.97$ & $70.38 \pm 0.94$ & $49.35 \pm 0.79$ \\
\hline \multirow{2}{*}{$2 b$} & $30 \mu \mathrm{M}$ & $76.33 \pm 1.79$ & $55.29 \pm 1.10$ & $83.32 \pm 1.96$ & $45.42 \pm 0.91$ \\
\hline & $100 \mu \mathrm{M}$ & $70.72 \pm 0.46$ & $53.8 \pm 1.41$ & $77.2 \pm 0.50$ & $43.31 \pm 2.63$ \\
\hline \multirow{2}{*}{$2 c$} & $30 \mu \mathrm{M}$ & $68.6 \pm 2.74$ & $72.09 \pm 2.30$ & $76.36 \pm 1.82$ & $59.22 \pm 1.89$ \\
\hline & $100 \mu \mathrm{M}$ & $59.84 \pm 2.20$ & $59.67 \pm 1.43$ & $65.32 \pm 2.40$ & $49.02 \pm 1.18$ \\
\hline \multirow{2}{*}{$2 d$} & $30 \mu \mathrm{M}$ & $19.34 \pm 2.54$ & $61.12 \pm 1.91$ & $21.11 \pm 2.78$ & $50.21 \pm 1.57$ \\
\hline & $100 \mu \mathrm{M}$ & $16.82 \pm 1.60$ & $24.55 \pm 1.85$ & $18.37 \pm 1.75$ & $20.17 \pm 1.52$ \\
\hline \multirow[b]{2}{*}{$2 e$} & $30 \mu \mathrm{M}$ & $67.73 \pm 1.34$ & $72.77 \pm 2.42$ & $73.94 \pm 1.46$ & $59.79 \pm 1.99$ \\
\hline & $100 \mu \mathrm{M}$ & $68.2 \pm 0.63$ & $61.01 \pm 1.16$ & $74.45 \pm 0.69$ & $50.12 \pm 0.95$ \\
\hline \multirow{2}{*}{$2 f$} & $30 \mu \mathrm{M}$ & $63.5 \pm 1.47$ & $69.84 \pm 1.85$ & $69.32 \pm 1.61$ & $57.38 \pm 1.52$ \\
\hline & $100 \mu \mathrm{M}$ & $54.53 \pm 1.19$ & $57.2 \pm 2.90$ & $59.53 \pm 1.30$ & $47.00 \pm 2.38$ \\
\hline \multirow{2}{*}{$2 g$} & $30 \mu \mathrm{M}$ & $76.83 \pm 2.31$ & $71.65 \pm 2.01$ & $83.87 \pm 2.52$ & $42.50 \pm 2.35$ \\
\hline & $100 \mu \mathrm{M}$ & $70.25 \pm 0.41$ & $47.9 \pm 2.03$ & $76.69 \pm 0.44$ & $39.35 \pm 1.67$ \\
\hline \multirow{2}{*}{$2 \mathrm{~h}$} & $30 \mu \mathrm{M}$ & $23.71 \pm 0.88$ & $44.42 \pm 2.35$ & $25.88 \pm 0.96$ & $36.50 \pm 1.93$ \\
\hline & $100 \mu \mathrm{M}$ & $21.86 \pm 0.20$ & $34.76 \pm 1.36$ & $23.86 \pm 0.22$ & $28.55 \pm 1.12$ \\
\hline \multirow{2}{*}{$2 \mathrm{i}$} & $30 \mu \mathrm{M}$ & $78.88 \pm 2.63$ & $64.37 \pm 1.47$ & $86.11 \pm 2.88$ & $52.89 \pm 1.21$ \\
\hline & $100 \mu \mathrm{M}$ & $76.06 \pm 0.27$ & $61.4 \pm 0.17$ & $83.03 \pm 0.29$ & $50.45 \pm 0.14$ \\
\hline \multirow{2}{*}{$2 j$} & $30 \mu \mathrm{M}$ & $65.01 \pm 2.17$ & $46.16 \pm 0.38$ & $70.97 \pm 2.37$ & $37.92 \pm 0.31$ \\
\hline & $100 \mu \mathrm{M}$ & $62.83 \pm 2.23$ & $40.66 \pm 1.04$ & $68.59 \pm 2.43$ & $33.40 \pm 0.86$ \\
\hline \multirow{2}{*}{$2 k$} & $30 \mu \mathrm{M}$ & $67.46 \pm 1.69$ & $69.88 \pm 2.12$ & $73.64 \pm 1.84$ & $57.41 \pm 1.74$ \\
\hline & $100 \mu \mathrm{M}$ & $46.78 \pm 0.21$ & $55.18 \pm 2.92$ & $51.06 \pm 0.23$ & $45.33 \pm 2.40$ \\
\hline \multirow{2}{*}{$3 a$} & $30 \mu \mathrm{M}$ & $72.06 \pm 1.92$ & $74.40 \pm 1.07$ & $78.67 \pm 2.09$ & $61.12 \pm 0.88$ \\
\hline & $100 \mu \mathrm{M}$ & $67.93 \pm 1.11$ & $69.77 \pm 0.35$ & $74.16 \pm 1.22$ & $57.32 \pm 0.29$ \\
\hline \multirow{2}{*}{$3 b$} & $30 \mu \mathrm{M}$ & $75.15 \pm 0.36$ & $70.17 \pm 1.90$ & $82.04 \pm 0.40$ & $57.64 \pm 1.56$ \\
\hline & $100 \mu \mathrm{M}$ & $71.76 \pm 0.48$ & $68.65 \pm 2.51$ & $78.34 \pm 0.52$ & $56.40 \pm 2.06$ \\
\hline
\end{tabular}


Table 4. Cont.

\begin{tabular}{cccccc}
\hline Comp. No. & Conc. & Hep3B & Hela & A549 & MCF-7 \\
\hline \multirow{2}{*}{ 3c } & $30 \mu \mathrm{M}$ & $85.76 \pm 2.42$ & $81.90 \pm 2.11$ & $93.62 \pm 2.64$ & $67.28 \pm 1.74$ \\
& $100 \mu \mathrm{M}$ & $68.37 \pm 1.58$ & $64.27 \pm 2.47$ & $74.63 \pm 1.73$ & $52.80 \pm 2.03$ \\
\hline \multirow{2}{*}{ 3d } & $30 \mu \mathrm{M}$ & $80.15 \pm 1.68$ & $81.46 \pm 1.60$ & $87.5 \pm 1.83$ & $66.92 \pm 1.31$ \\
& $100 \mu \mathrm{M}$ & $72.57 \pm 1.83$ & $73.28 \pm 2.50$ & $79.22 \pm 2.00$ & $60.20 \pm 2.05$ \\
\hline \multirow{2}{*}{ 3e } & $30 \mu \mathrm{M}$ & $67.02 \pm 1.37$ & $80.59 \pm 1.39$ & $73.17 \pm 1.49$ & $66.21 \pm 1.14$ \\
\hline \multirow{2}{*}{ 3f } & $100 \mu \mathrm{M}$ & $53.79 \pm 0.71$ & $77.66 \pm 0.29$ & $58.72 \pm 0.77$ & $63.80 \pm 0.24$ \\
& $30 \mu \mathrm{M}$ & $72.26 \pm 1.01$ & $77.05 \pm 2.19$ & $78.89 \pm 1.11$ & $63.30 \pm 1.80$ \\
\multirow{2}{*}{ 3g } & $100 \mu \mathrm{M}$ & $65.95 \pm 0.25$ & $67.78 \pm 1.64$ & $71.99 \pm 0.28$ & $55.68 \pm 1.35$ \\
\hline \multirow{2}{*}{ 3h } & $30 \mu \mathrm{M}$ & $80.42 \pm 1.16$ & $62.38 \pm 0.71$ & $87.79 \pm 1.27$ & $51.25 \pm 0.58$ \\
& $100 \mu \mathrm{M}$ & $70.65 \pm 1.77$ & $51.23 \pm 0.49$ & $77.13 \pm 1.94$ & $42.09 \pm 0.40$ \\
\hline \multirow{2}{*}{ 3i } & $30 \mu \mathrm{M}$ & $75.08 \pm 1.11$ & $81.90 \pm 1.04$ & $81.96 \pm 1.21$ & $67.28 \pm 0.85$ \\
& $100 \mu \mathrm{M}$ & $67.16 \pm 2.57$ & $75.92 \pm 1.60$ & $73.31 \pm 2.81$ & $62.37 \pm 1.31$ \\
\hline \multirow{2}{*}{ 3j } & $30 \mu \mathrm{M}$ & $78.34 \pm 0.71$ & $77.12 \pm 2.03$ & $85.52 \pm 0.77$ & $63.36 \pm 1.67$ \\
& $100 \mu \mathrm{M}$ & $74.04 \pm 0.61$ & $56.81 \pm 1.81$ & $80.83 \pm 0.67$ & $46.67 \pm 1.49$ \\
\hline \multirow{2}{*}{ CPT * } & $30 \mu \mathrm{M}$ & $69.54 \pm 2.39$ & $87.18 \pm 1.91$ & $75.92 \pm 2.61$ & $71.62 \pm 1.57$ \\
& $100 \mu \mathrm{M}$ & $61.25 \pm 2.24$ & $74.51 \pm 2.17$ & $66.86 \pm 2.45$ & $61.21 \pm 1.78$ \\
\hline \multirow{2}{*}{$0.3 \mu \mathrm{M}$} & $69.56 \pm 1.27$ & $57.06 \pm 1.35$ & $67.68 \pm 1.88$ & $56.68 \pm 0.68$ \\
& * Camptothecin. Data is presented as percentage of the cell viability $\pm \mathrm{SD}$. & \\
\hline
\end{tabular}

\section{Experimental}

\subsection{General Information}

In this study, 4-(1-adamantyl)-3-thiosemicarbazide (1) was synthesized by the reaction of adamantan-1-yl isothiocyanate (Sigma Aldrich, MO, USA) with 80\% hydrazine hydrate solution (Sigma Aldrich, St. Louis, MO, USA) in toluene under microwave irradiated conditions for $30 \mathrm{~min}$ at $100 \mathrm{~W}$ following the same method as a previous publication [47]. Common chemicals such as methanol $(\mathrm{MeOH})$, acetic acid (AcOH), substituted benzaldehydes and acetophenones were also supplied by Sigma Aldrich (St. Louis, MO, USA), Serva Electrophoresis GmbH (Heidelberg, Germany), Fisher Sciencetific (Loughborough, UK), Acros Organics (Branchburg, NJ, USA); and used directly without further purification.

Melting points $\left({ }^{\circ} \mathrm{C}\right)$ were checked in a micro tube glass with an electrothermal melting point apparatus (MP50 Mettler Toledo, Columbus, OH, USA). NMR spectra data was recorded at $500 \mathrm{MHz}$ in DMSO- $d_{6}$ using an AVANCE III spectrometer (Bruker Biospin, Billerica, MA, USA); the chemical shifts $(\delta, \mathrm{ppm})$ were expressed and coupling constants $(J)$ were given in $\mathrm{Hz}$ using the internal standard tetramethylsilane. Mass spectra (MS) was obtained on a 910-TQ-FT-MS system, (Agilent, Santa Clara, CA, USA). Processing the reactions and preliminary purity evaluating of synthesized compounds were verified by thin layer chromatography (TLC, pre-coated aluminum sheet 60 F254 plates, Merck KGaA Co., Darmstadt, Germany) and visualization at UV $254 \mathrm{~nm}$. The bacterial and fungus strains, i.e., Escherichia coli ATCC25922, Pseudomonas aeruginosa ATCC27853, Salmonella enterica ATCC12228, Enterococcus faecalis ATCC13124, Stapphylococus aureus ATCC25923, Bacillus cereus ATCC 13245, and Candida albicans ATCC10231 were purchased by National Institute for Food Control (NIFC, Hanoi, Vietnam). This study was conducted on cancer cell lines supplied by Advanced Center for Bioorganic Chemistry (ACBC) of the Institute of Marine Biochemistry (IMBC), Vietnam Academy Science and Technology (VAST), i.e., hepatic cancer cell line Hep3B, human cervical cancer cell line HeLa, human lung cancer cell line A549, and human breast carcinoma MCF-7. 


\subsection{Synthesis of Thiosemicarbazones $\mathbf{2} \mathbf{a}-\mathbf{k}$ and $\mathbf{3 a} \mathbf{a}-\mathbf{j}$}

A mixture of $1 \mathrm{mmol}$ of 4-(1-adamantyl)-3-thiosemicarbazide (1) and $3 \mathrm{mmol}$ of the appropriate aldehyde or ketone and $20 \mathrm{~mL} \mathrm{MeOH}$ was put in a three necked glass round bottomed flask and mildly heated. Afterwards glacial acetic acid was dropped into this solution to adjust the $\mathrm{pH}$ to 4-5, and the reaction mixture was refluxed. The reaction was monitored by TLC (chloroform/aceton, 95/5, $\mathrm{v} / \mathrm{v}$ ), and visualized under UV at $254 \mathrm{~nm}$ and Dragendorff reagent. The reaction was continued until 4-(1-adamantyl)-3-thiosemicarbazide (1) was completely consumed. After the reaction was complete, the reaction mixture was evaporated to yield a solid which was washed with ice-cold $\mathrm{MeOH}$ to remove residual aldehydes or ketones, and recrystallized from $\mathrm{MeOH}$. The crystals were dried below $50{ }^{\circ} \mathrm{C}$ to obtain thiosemicarbazones $\mathbf{2} \mathbf{a}-\mathbf{k}$ and $\mathbf{3 a}-\mathbf{j}$.

4-(N-Adamantan-1-yl)-1-(1-benzylidene)thiosemicarbazone (2a): ${ }^{1} \mathrm{H}-\mathrm{NMR}(\delta \mathrm{ppm}): 11.29(1 \mathrm{H}, \mathrm{s},-\mathrm{CS}-\mathrm{NH}-\mathrm{N})$; $8.08(1 \mathrm{H}, \mathrm{s}, \mathrm{N}=\mathrm{CH}) ; 7.66(2 \mathrm{H}, \mathrm{dd}, \mathrm{J} 1=2.0 \mathrm{~Hz}, \mathrm{~J} 2=7.5 \mathrm{~Hz}, \mathrm{Ar}-\mathrm{H}) ; 7.49$ (1H, s, C-NH-CS); 7.44-7.41 (3H, $\mathrm{m}, \mathrm{Ar}-\mathrm{H}) ; 2.28(6 \mathrm{H}, \mathrm{m}$, adamantane-H); $2.08(3 \mathrm{H}, \mathrm{m}$, adamantane-H); $1.66(6 \mathrm{H}, \mathrm{m}$, adamantane-H); ${ }^{13} \mathrm{C}-\mathrm{NMR}(\delta \mathrm{ppm}): 174.6(1 \mathrm{C}, \mathrm{CS}) ; 141.4(1 \mathrm{C}, \mathrm{CH}=\mathrm{N}) ; 133.8(1 \mathrm{C}, \mathrm{Ar}-\mathrm{C}) ; 129.9$ (1C, Ar-C); $128.8(2 \mathrm{C}$, Ar-C); 126.9 (2C, Ar-C); 53.0 (1C, C-N); 40.9 (3C, Adamantane-C); 35.9 (3C, Adamantane-C); 29.0 (3C, Adamantane-C). ESI-HR-MS $(m / z):[\mathrm{M}+\mathrm{H}]^{+}=314.1676$.

4-(N-Adamantan-1-yl)-1-[1-(3-nitrobenzylidene)] thiosemicarbazone (2b): ${ }^{1} \mathrm{H}-\mathrm{NMR}(\delta \mathrm{ppm}): 11.50(1 \mathrm{H}, \mathrm{s}$, -CS-NH-N); $8.43(1 \mathrm{H}, \mathrm{t}, J 1=1.5 \mathrm{~Hz}, \mathrm{~J} 2=8.5 \mathrm{~Hz}, \mathrm{~N}=\mathrm{CH}) ; 8.23-8.21(1 \mathrm{H}, \mathrm{dd}, J 1=2.0 \mathrm{~Hz}, \mathrm{~J} 2=7.5 \mathrm{~Hz}, \mathrm{Ar}-\mathrm{H})$; 8.18-8.15 (2H, m, Ar-H); 7.73-7.70 (1H, t, J1 = 8.0 Hz, J2 = 16.0 Hz, Ar-H); 7.56 (1H, s, C-NH-CS); 2.29 $\left(6 \mathrm{H}, \mathrm{m}\right.$, adamantane-H); $2.09\left(3 \mathrm{H}, \mathrm{m}\right.$, adamantane-H); $1.67\left(6 \mathrm{H}, \mathrm{m}\right.$, adamantane-H). ${ }^{13} \mathrm{C}-\mathrm{NMR}(\delta \mathrm{ppm})$ : 174.8 (1C, CS); 148.2 (1C, Ar-C); 139.0 (1C, CH=N); 135.8 (1C, Ar-C); 132.7 (1C, Ar-C); 130.3 (1C, Ar-C); 124.0 (1C, Ar-C); 121.3 (1C, Ar-C); 53.2 (1C, C-N); 40.7 (3C, adamantane-C); 35.9 (3C, adamantane-C); $29.0\left(3 \mathrm{C}\right.$, adamantane-C). ESI-MS $(m / z):[\mathrm{M}+\mathrm{H}]^{+}=358.9 ;[\mathrm{M}-\mathrm{H}]^{-}=356.9$.

4-(N-Adamantan-1-yl)-1-[1-(4-methoxybenzylidene)]thiosemicarbazone (2c): ${ }^{1} \mathrm{H}-\mathrm{NMR}(\delta \mathrm{ppm}): 11.16(1 \mathrm{H}, \mathrm{s}$, -CS-NH-N); 8.02 (1H, s, N=CH); 7.62-7.60 (2H, d, J = 9.0 Hz, Ar-H); 7.44 (1H, s, C-NH-CS); 6.99-6.97 $(2 \mathrm{H}, \mathrm{d}, \mathrm{J}=8.5 \mathrm{~Hz}, \mathrm{Ar}-\mathrm{H}) ; 3.79\left(3 \mathrm{H}, \mathrm{s}, \mathrm{OCH}_{3}\right) ; 2.27(6 \mathrm{H}, \mathrm{m}$, adamantane-H); $2.08(3 \mathrm{H}, \mathrm{m}$, adamantane-H); $1.67\left(6 \mathrm{H}, \mathrm{m}\right.$, adamantane-H). ${ }^{13} \mathrm{C}-\mathrm{NMR}(\delta \mathrm{ppm}): 174.4(1 \mathrm{C}, \mathrm{CS}) ; 160.7(1 \mathrm{C}, \mathrm{C} 4) ; 141.4(1 \mathrm{C}, \mathrm{CH}=\mathrm{N}) ; 128.5$ (2C, Ar-C); 126.4 (1C, Ar-C); 114.3 (2C, Ar-C); 55.2 (1C, $\left.\mathrm{OCH}_{3}\right) ; 52.9$ (1C, C-N); 41.0 (3C, adamantane-C); 35.9 (3C, adamantane-C); 29.0 (3C, adamantane-C). ESI-MS $[\mathrm{m} / \mathrm{z}]:[\mathrm{M}+\mathrm{H}]^{+}=343.9 ;[\mathrm{M}-\mathrm{H}]^{-}=341.9$.

4-(N-Adamantan-1-yl)-1-[1-(2-hydroxybenzylidene)]thiosemicarbazone (2d): ${ }^{1} \mathrm{H}-\mathrm{NMR}(\delta \mathrm{ppm}): 11.23(1 \mathrm{H}, \mathrm{s}$, -CS-NH-N); $9.97(1 \mathrm{H}, \mathrm{s}, \mathrm{OH}) ; 8.37(1 \mathrm{H}, \mathrm{s}, \mathrm{N}=\mathrm{CH}) ; 7.67(1 \mathrm{H}, \mathrm{d}, J=7.5 \mathrm{~Hz}, \mathrm{Ar}-\mathrm{H}) ; 7.46$ (1H, s, C-NH-CS); 7.23-7.20 (1H, t, J1 = 7.5Hz; J2 = $15.0 \mathrm{~Hz}, \mathrm{Ar}-\mathrm{H})$; 6.88-6.87 (1H, d, J = 7.5 Hz, Ar-H); 6.85-6.82 (1H, t, J1 $=7.5 \mathrm{~Hz} ; \mathrm{J} 2=15.0 \mathrm{~Hz}, \mathrm{Ar}-\mathrm{H}) ; 2.27(6 \mathrm{H}, \mathrm{m}$, adamantane-H); $2.07(3 \mathrm{H}, \mathrm{m}$, adamantane-H); $1.66(6 \mathrm{H}$, $\mathrm{m}$, adamantane-H). ${ }^{13} \mathrm{C}-\mathrm{NMR}(\delta \mathrm{ppm}): 174.5(1 \mathrm{C}, \mathrm{CS}) ; 156.5(1 \mathrm{C}, \mathrm{Ar}-\mathrm{C}) ; 138.5(1 \mathrm{C}, \mathrm{CH}=\mathrm{N}) ; 131.0(1 \mathrm{C}$, Ar-C); 125.9 (1C, Ar-C); 120.3 (1C, Ar-C); 119.3 (1C, Ar-C); 116.1 (1C, Ar-C); 52.9 (1C, C-N); 41.0 (3C, adamantane-C); 35.9 (3C, adamantane-C); 29.0 (3C, adamantane-C). ESI-MS [m/z]: $[\mathrm{M}+\mathrm{H}]^{+}=330.0$; $[\mathrm{M}-\mathrm{H}]^{-}=327.9$.

4-(N-Adamantan-1-yl)-1-[1-(4-nitrobenzylidene)]thiosemicarbazone (2e): ${ }^{1} \mathrm{H}-\mathrm{NMR}(\delta \mathrm{ppm}): 11.56(1 \mathrm{H}, \mathrm{s}$, -CS-NH-N); 8.25-8.23 (2H, d, J = 8.5 Hz, Ar-H); $8.15(1 \mathrm{H}, \mathrm{s}, \mathrm{N}=\mathrm{CH}) ; 7.95-7.93(2 \mathrm{H}, \mathrm{d}, J=9.0 \mathrm{~Hz}$, Ar-H); 7.57 (1H, s, C-NH); 2.29 (6H, m, adamantane-H); 2.09 (3H, m, adamantane-H); $1.66(6 \mathrm{H}, \mathrm{m}$, adamantane-H). ${ }^{13} \mathrm{C}-\mathrm{NMR}(\delta \mathrm{ppm}): 174.8(1 \mathrm{C}, \mathrm{CS}) ; 147.6(1 \mathrm{C}, \mathrm{Ar}-\mathrm{C}) ; 140.3(1 \mathrm{C}, \mathrm{CH}=\mathrm{N}) ; 138.8(1 \mathrm{C}, \mathrm{Ar}-\mathrm{C})$; 127.8 (2C, Ar-C); 123.9 (2C, Ar-C); 53.3 (C-NH); 40.7 (3C, adamantane-C); 35.9 (3C, adamantane-C); 29.0 (3C, adamantane-C). ESI-MS $[\mathrm{m} / \mathrm{z}]:[\mathrm{M}+\mathrm{H}]^{+}=358.9 ;[\mathrm{M}-\mathrm{H}]^{-}=356.9$.

4-(N-Adamantan-1-yl)-1-[1-(4-ethoxybenzylidene)]thiosemicarbazone (2f): ${ }^{1} \mathrm{H}-\mathrm{NMR}(\delta \mathrm{ppm}): 11.16(1 \mathrm{H}$, s, -CS-NH-N); 8.01 (1H, s, N=CH); 7.60-7.58 (2H, d, J = 8.6 Hz, Ar-H); 7.43 (1H, s, C-NH); 6.97-6.95 $(2 \mathrm{H}, \mathrm{d}, \mathrm{J}=8.5 ; \mathrm{Ar}-\mathrm{H}) ; 4.07-4.05\left(2 \mathrm{H}, \mathrm{d}, \mathrm{J}=7.0 \mathrm{~Hz}, \mathrm{OCH}_{2}\right) ; 2.27(6 \mathrm{H}, \mathrm{m}$, adamantane-H); $2.08(3 \mathrm{H}, \mathrm{m}$, adamantane-H); $1.66\left(6 \mathrm{H}, \mathrm{m}\right.$, adamantane-H); $1.35-1.32\left(3 \mathrm{H}, \mathrm{t}, \mathrm{J} 1=7.0 \mathrm{~Hz}, \mathrm{~J} 2=14.0 \mathrm{~Hz}, \mathrm{CH}_{2}-\mathrm{CH}_{3}\right)$. 
${ }^{13} \mathrm{C}-\mathrm{NMR}(\delta \mathrm{ppm}): 174.4(1 \mathrm{C}, \mathrm{CS}) ; 160.0$ (1C, Ar-C); 141.5 (1C, CH=N); 128.5 (2C, Ar-C); 126.2 (1C, Ar-C); 114.7 (2C, Ar-C); $63.2\left(1 \mathrm{C}, \mathrm{OCH}_{2}\right) ; 52.8(\mathrm{C}-\mathrm{NH}) ; 41.0$ (3C, adamantane-C); 35.9 (3C, adamantane-C); 29.0 (3C, adamantane-C); $14.5\left(1 \mathrm{C}, \mathrm{CH}_{2} \mathrm{CH}_{3}\right)$. ESI-MS $[\mathrm{m} / \mathrm{z}]:[\mathrm{M}+\mathrm{H}]^{+}=358.0 ;[\mathrm{M}-\mathrm{H}]^{-}=355.9$.

4-(N-Adamantan-1-yl)-1-[1-(4-chlorobenzylidene)] thiosemicarbazone (2g): ${ }^{1} \mathrm{H}-\mathrm{NMR}(\delta \mathrm{ppm}): 11.34(1 \mathrm{H}, \mathrm{s}$, -CS-NH-N); $8.05(1 \mathrm{H}, \mathrm{s}, \mathrm{N}=\mathrm{CH}) ; 7.70(2 \mathrm{H}, \mathrm{d}, J=8.5 \mathrm{~Hz}, \mathrm{Ar}-\mathrm{H}) ; 7.48-7.47$ (3H, m, Ar-H \& NH-C); 2.28 $(6 \mathrm{H}, \mathrm{m}$, adamantane- $\mathrm{H}) ; 2.08(3 \mathrm{H}, \mathrm{m}$, adamantane- $\mathrm{H}) ; 1.66(6 \mathrm{H}, \mathrm{m}$, adamantane- $\mathrm{H}) .{ }^{13} \mathrm{C}-\mathrm{NMR}(\delta \mathrm{ppm})$ : 174.7 (1C, CS); 140.1 (1C, CH=N); 134.3 (1C, Ar-C); 132.8 (1C, Ar-C); 128.8 (2C, Ar-C); 128.6 (2C, Ar-C); 53.1 (C-NH); 40.9 (3C, adamantane-C); 35.9 (3C, adamantane-C); 29.0 (3C, adamantane-C). ESI-MS $[\mathrm{m} / \mathrm{z}]:[\mathrm{M}+\mathrm{H}]^{+}=347.9 ;[\mathrm{M}-\mathrm{H}]^{-}=345.9$.

4-(N-Adamantan-1-yl)-1-[1-(2-hydroxy-5-methylbenzylidene)]thiosemicarbazone (2h): ${ }^{1} \mathrm{H}-\mathrm{NMR}(\delta \mathrm{ppm})$ : $11.20(1 \mathrm{H}, \mathrm{s},-\mathrm{CS}-\mathrm{NH}-\mathrm{N}) ; 9.73(1 \mathrm{H}, \mathrm{s}, \mathrm{OH}) ; 8.34(1 \mathrm{H}, \mathrm{s}, \mathrm{N}=\mathrm{CH}) ; 7.45(1 \mathrm{H}, \mathrm{s}, \mathrm{C}-\mathrm{NH}) ; 7.44(1 \mathrm{H}, \mathrm{s}, \mathrm{Ar}-\mathrm{H})$; 7.04-7.02 $(1 \mathrm{H}, \mathrm{dd}, J 1=2.0 \mathrm{~Hz}, J 2=8.5 \mathrm{~Hz}, \mathrm{Ar}-\mathrm{H}) ; 6.78-6.76(1 \mathrm{H}, \mathrm{d}, J=7.5 \mathrm{~Hz}, \mathrm{Ar}-\mathrm{H}) ; 2.27(6 \mathrm{H}$, $\mathrm{m}$, adamantane- $\mathrm{H}) ; 2.21\left(3 \mathrm{H}, \mathrm{s}, \mathrm{CH}_{3}\right) ; 2.08(3 \mathrm{H}, \mathrm{m}$, adamantane- $\mathrm{H}) ; 1.66(6 \mathrm{H}, \mathrm{m}$, adamantane- $\mathrm{H})$. ${ }^{13} \mathrm{C}-\mathrm{NMR}(\delta \mathrm{ppm}): 174.5$ (1C, CS); 154.4 (1C, Ar-C); 138.9 (1C, CH=N); 131.8 (1C, Ar-C); 127.8 (1C, Ar-C); 125.9 (1C, Ar-C); 119.8 (1C, Ar-C); 116.0 (1C, Ar-C); 52.8 (C-NH); 40.9 (3C, adamantane-C); 35.9 (3C, adamantane-C); 29.0 (3C, adamantane-C); 20.1 (1C, $\left.\mathrm{CH}_{3}\right)$. ESI-MS [m/z]: $[\mathrm{M}+\mathrm{H}]^{+}=344.0$; $[\mathrm{M}-$ $\mathrm{H}]^{-}=341.9$.

4-(N-Adamantan-1-yl)-1-[1-(3-nitro-4-ethoxybenzylidene)]thiosemicarbazone (2i): ${ }^{1} \mathrm{H}-\mathrm{NMR}(\delta \mathrm{ppm}): 11.34$ $(1 \mathrm{H}, \mathrm{s},-\mathrm{CS}-\mathrm{NH}-\mathrm{N}) ; 8.16-8.15(1 \mathrm{H}, \mathrm{d}, J=2.5 \mathrm{~Hz}, \mathrm{Ar}-\mathrm{H}) ; 8.05(1 \mathrm{H}, \mathrm{s}, \mathrm{N}=\mathrm{CH}) ; 7.93-7.91(1 \mathrm{H}, \mathrm{dd}, J 1=2.0$ $\mathrm{Hz}, J 2=8.5 \mathrm{~Hz}, \mathrm{Ar}-\mathrm{H}) ; 7.48(1 \mathrm{H}, \mathrm{s}, \mathrm{C}-\mathrm{NH}) ; 7.39-7.37(1 \mathrm{H}, \mathrm{d}, J=8.5 \mathrm{~Hz}, \mathrm{Ar}-\mathrm{H}) ; 4.28-4.24(2 \mathrm{H}, \mathrm{dd}, J 1=$ $\left.7.0 \mathrm{~Hz} ; \mathrm{J} 2=14.0 \mathrm{~Hz}, \mathrm{OCH}_{2}\right) ; 2.28(6 \mathrm{H}, \mathrm{m}$, adamantane-H); $2.08(3 \mathrm{H}, \mathrm{m}$, adamantane-H); $1.66(6 \mathrm{H}, \mathrm{m}$, adamantane-H); 1.36-1.33 (3H, t, J1 = 7.0; $\left.J 2=14.0 \mathrm{~Hz}, \mathrm{CH}_{3}\right) .{ }^{13} \mathrm{C}-\mathrm{NMR}(\delta \mathrm{ppm}): 174.6(1 \mathrm{C}, \mathrm{CS}) ; 151.7$ (1C, Ar-C); $140.0(1 \mathrm{C}, \mathrm{CH}=\mathrm{N}) ; 139.2$ (1C, Ar-C); 132.2 (1C, Ar-C); 126.5 (1C, Ar-C); 122.8 (1C, Ar-C); 115.3 (1C, Ar-C); $65.3\left(1 \mathrm{C}, \mathrm{OCH}_{2}\right) ; 53.1(\mathrm{C}-\mathrm{NH}) ; 40.8$ (3C, adamantane-C); 35.9 (3C, adamantane-C); 29.0 (3C, adamantane-C); $14.2\left(1 \mathrm{C}, \mathrm{CH}_{3}\right)$. ESI-MS $[\mathrm{m} / \mathrm{z}]:[\mathrm{M}+\mathrm{H}]^{+}=403.0 ;[\mathrm{M}-\mathrm{H}]^{-}=400.9$.

4-(N-Adamantan-1-yl)-1-[1-(3-nitro-4-chlorobenzylidene)] thiosemicarbazone (2j): ${ }^{1} \mathrm{H}-\mathrm{NMR}(\delta \mathrm{ppm}): 11.53$ $(1 \mathrm{H}, \mathrm{s},-\mathrm{CS}-\mathrm{NH}-\mathrm{N}) ; 8.36(1 \mathrm{H}, \mathrm{d}, J=1.0 \mathrm{~Hz}, \mathrm{~N}=\mathrm{CH}) ; 8.09(1 \mathrm{H}, \mathrm{s}, \mathrm{Ar}-\mathrm{H}) ; 8.0(1 \mathrm{H}, \mathrm{d}, J=8.0 \mathrm{~Hz}, \mathrm{Ar}-\mathrm{H}) ; 7.79$ $(1 \mathrm{H}, \mathrm{d}, J=8.5 \mathrm{~Hz}, \mathrm{Ar}-\mathrm{H}) ; 7.54(1 \mathrm{H}, \mathrm{s}, \mathrm{NH}-\mathrm{C}) ; 2.28(6 \mathrm{H}, \mathrm{m}$, adamantane- $\mathrm{H}) ; 2.08(3 \mathrm{H}, \mathrm{m}$, adamantane- $\mathrm{H})$; $1.66\left(6 \mathrm{H}, \mathrm{m}\right.$, adamantane-H). ${ }^{13} \mathrm{C}-\mathrm{NMR}(\delta \mathrm{ppm}): 174.8(1 \mathrm{C}, \mathrm{CS}) ; 148.1(1 \mathrm{C}, \mathrm{Ar}-\mathrm{C}) ; 137.9(1 \mathrm{C}, \mathrm{CH}=\mathrm{N})$; 134.7 (1C, Ar-C); 131.8 (1C, Ar-C); 131.3 (1C, Ar-C); 125.0 (1C, Ar-C); 123.2 (1C, Ar-C); 53.3 (C-NH); 40.7 (3C, adamantane-C); 35.9 (3C, adamantane-C); 29.0 (3C, adamantane-C). ESI-MS [ $m / z]:[\mathrm{M}+\mathrm{H}]^{+}=$ 392.9; $[\mathrm{M}-\mathrm{H}]^{-}=390.9$.

4-(N-Adamantan-1-yl)-1-[1-(2,5-dimethylbenzylidene)]thiosemicarbazone (2k): ${ }^{1} \mathrm{H}-\mathrm{NMR}(\delta \mathrm{ppm}): 11.21(1 \mathrm{H}$, s, -CS-NH-N); 8.33 (1H, s, N=CH); 7.49 (1H, s, Ar-H); 7.42 (1H, NH-C); 7.12-7.11 (2H, m, Ar-H); 2.35 $\left(3 \mathrm{H}, \mathrm{s}, \mathrm{CH}_{3}\right) ; 2.29\left(3 \mathrm{H}, \mathrm{s}, \mathrm{CH}_{3}\right) ; 2.27(6 \mathrm{H}, \mathrm{m}$, adamantane- $\mathrm{H}) ; 2.07(3 \mathrm{H}, \mathrm{m}$, adamantane- $\mathrm{H}) ; 1.66(6 \mathrm{H}$, $\mathrm{m}$, adamantane-H). ${ }^{13} \mathrm{C}-\mathrm{NMR}(\delta \mathrm{ppm}): 174.5(1 \mathrm{C}, \mathrm{CS}) ; 141.2(1 \mathrm{C}, \mathrm{CH}=\mathrm{N}) ; 135.1(1 \mathrm{C}, \mathrm{Ar}-\mathrm{C}) ; 133.7(1 \mathrm{C}$, Ar-C); 131.5 (1C, Ar-C); 130.9 (1C, Ar-C); 130.3 (1C, Ar-C); 127.0 (1C, Ar-C); 52.9 (C-NH); 40.9 (3C, adamantane-C); 35.8 (3C, adamantane-C); 29.0 (3C, adamantane-C); 20.4 (1C, $\left.\mathrm{CH}_{3}\right) ; 19.2\left(1 \mathrm{C}, \mathrm{CH}_{3}\right)$. ESI-MS $[\mathrm{m} / \mathrm{z}]:[\mathrm{M}+\mathrm{H}]^{+}=342.0 ;[\mathrm{M}-\mathrm{H}]^{-}=340.0$.

4-(N-Adamantan-1-yl)-1-(1-phenylethylidene)thiosemicarbazone (3a): ${ }^{1} \mathrm{H}-\mathrm{NMR}(\delta \mathrm{ppm}): 9.77(1 \mathrm{H}, \mathrm{s}$, -CS-NH-N); $7.69(2 \mathrm{H}, \mathrm{d}, J=8.5 \mathrm{~Hz}, \mathrm{Ar}-\mathrm{H}) ; 7.63(1 \mathrm{H}, \mathrm{s}, \mathrm{Ar}-\mathrm{H}) ; 7.59(2 \mathrm{H}, \mathrm{d}, J=8.5 \mathrm{~Hz}, \mathrm{Ar}-\mathrm{H})$; $2.30\left(3 \mathrm{H}, \mathrm{s}, \mathrm{CH}_{3}\right) ; 2.28(6 \mathrm{H}, \mathrm{s}$, adamantane-H); $2.09(3 \mathrm{H}, \mathrm{s}$, adamantane- $\mathrm{H}) ; 1.68(6 \mathrm{H}, \mathrm{s}$, adamantane- $\mathrm{H})$. ${ }^{13} \mathrm{C}-\mathrm{NMR}(\delta \mathrm{ppm}): 175.5$ (1C, CS); 147.1 (1C, N=C); 137.7 (1C, Ar-C); 129.2 (1C, Ar-C); 128.4 (2C, Ar-C); 126.0 (2C, Ar-C); 52.9 (C-NH); 40.8 (3C, adamantane-C); 35.8 (3C, adamantane-C); 28.9 (3C, adamantane-C); $14.2\left(1 \mathrm{C}, \mathrm{CH}_{3}\right)$. ESI-MS $[\mathrm{m} / \mathrm{z}]:[\mathrm{M}+\mathrm{H}]^{+}=325.9 ;[\mathrm{M}-\mathrm{H}]^{-}=328.0$.

4-(N-Adamantan-1-yl)-1-[1-(3-nitrophenyl)ethylidene]thiosemicarbazone (3b): ${ }^{1} \mathrm{H}-\mathrm{NMR}(\delta \mathrm{ppm}): 10.22(1 \mathrm{H}$, s, -CS-NH-N); 8.49 (1H, s, Ar-H); $8.23(1 \mathrm{H}, \mathrm{dd}, J 1=1.5 \mathrm{~Hz}, \mathrm{~J} 2=8.0 \mathrm{~Hz}, \mathrm{Ar}-\mathrm{H}) ; 8.19(1 \mathrm{H}, \mathrm{d}, J=8.0 \mathrm{~Hz}$, 
Ar-H); $7.73(1 \mathrm{H}, \mathrm{s}, \mathrm{NH}-\mathrm{C}) ; 7.70(1 \mathrm{H}, \mathrm{d}, J=8.0 \mathrm{~Hz}, \mathrm{Ar}-\mathrm{H}) ; 2.37\left(3 \mathrm{H}, \mathrm{s}, \mathrm{CH}_{3}\right) ; 2.28(6 \mathrm{H}$, brs, adamantane-H); 2.08 (3H, brs, adamantane-H); 1.66 (6H, brs, adamantane-H). ${ }^{13} \mathrm{C}-\mathrm{NMR}(\delta \mathrm{ppm}): 175.7$ (1C, CS); 148.1 (1C, N=C); 144.8 (1C, Ar-C); 139.5 (1C, Ar-C); 132.4 (1C, Ar-C); 130.2 (1C, Ar-C); 123.6 (1C; Ar-C); 120.5 (1C; Ar-C); 53.2 (C-NH); 40.8 (3C, adamantane-C); 35.9 (3C, adamantane-C); 29.0 (3C, adamantane-C); $14.3\left(1 \mathrm{C}, \mathrm{CH}_{3}\right)$. ESI-MS $[\mathrm{m} / \mathrm{z}]:[\mathrm{M}+\mathrm{H}]^{+}=372.9 ;[\mathrm{M}-\mathrm{H}]^{-}=370.9$.

4-(N-Adamantan-1-yl)-1-[1-(4-bromophenyl)ethylidene]thiosemicarbazone (3c): ${ }^{1} \mathrm{H}-\mathrm{NMR}(\delta \mathrm{ppm}): 9.77(1 \mathrm{H}$, s, -CS-NH-N); 7.70-7.68 (2H, d, J = 8.5 Hz, Ar-H); $7.63(1 \mathrm{H}, \mathrm{s}, \mathrm{NH}-\mathrm{C}) ; 7.60-7.59(2 \mathrm{H}, \mathrm{d}, J=8.5 \mathrm{~Hz}$, $\mathrm{Ar}-\mathrm{H}) ; 2.29\left(3 \mathrm{H}, \mathrm{s}, \mathrm{CH}_{3}\right) ; 2.28\left(3 \mathrm{H}, \mathrm{s}, \mathrm{CH}_{3}\right) ; 2.09(3 \mathrm{H}, \mathrm{m}$, adamantane-H); $1.68(6 \mathrm{H}, \mathrm{m}$, adamantane-H). ${ }^{13} \mathrm{C}-\mathrm{NMR}(\delta \mathrm{ppm}): 175.5$ (1C, CS); $146.2(1 \mathrm{C}, \mathrm{C}=\mathrm{N}) ; 136.9$ (1C, Ar-C); $131.4(2 \mathrm{C}, \mathrm{Ar}-\mathrm{C}) ; 128.2(2 \mathrm{C}$, Ar-C); 122.7 (1C, Ar-C); 53.1 (C-NH); 40.8 (3C, adamantane-C); 35.9 (3C, adamantane-C); 29.0 (3C, adamantane-C); $14.1\left(1 \mathrm{C}, \mathrm{CH}_{3}\right)$. ESI-MS $[\mathrm{m} / \mathrm{z}]$ : $[\mathrm{M}+\mathrm{H}]^{+}=407.9 ;[\mathrm{M}-\mathrm{H}]^{-}=405.8$.

4-(N-Adamantan-1-yl)-1-[1-(4-hydroxyphenyl)ethylidene]thiosemicarbazone (3d): ${ }^{1} \mathrm{H}-\mathrm{NMR}(\delta \mathrm{ppm}): 9.83$ $(1 \mathrm{H}, \mathrm{s},-\mathrm{CS}-\mathrm{NH}-\mathrm{N}) ; 7.64(1 \mathrm{H}, \mathrm{s}, \mathrm{NH}-\mathrm{C}) ; 7.59$ (2H, d, J = 8.5 Hz, Ar-H); $6.79(2 \mathrm{H}, \mathrm{d}, J=9.0 \mathrm{~Hz}, \mathrm{Ar}-\mathrm{H}) ; 2.26$ (6H, brs, adamantane- $\mathrm{H}) ; 2.24\left(3 \mathrm{H}, \mathrm{s}, \mathrm{CH}_{3}\right) ; 2.07$ (3H, brs, adamantane- $\left.\mathrm{H}\right) ; 1.65(6 \mathrm{H}$, brs, adamantane- $\mathrm{H})$. ${ }^{13} \mathrm{C}-\mathrm{NMR}(\delta \mathrm{ppm}): 175.3$ (1C, CS); 158.8 (1C, Ar-C), 147.7 (1C, N=C); 128.4 (1C, Ar-C); 127.7 (2C, Ar-C); 115.3 (2C, Ar-C); 52.9 (C-NH); 41.0 (3C, adamantane-C); 35.9 (3C, adamantane-C); 29.0 (3C, adamantane-C); $14.1\left(1 \mathrm{C}, \mathrm{CH}_{3}\right)$. ESI-MS $[\mathrm{m} / \mathrm{z}]:[\mathrm{M}+\mathrm{H}]^{+}=344.0 ;[\mathrm{M}-\mathrm{H}]^{-}=341.9$.

4-(N-adamantan-1-yl)-1-[1-(4-nitro-phenyl)ethylidene]thiosemicarbazone (3e): ${ }^{1} \mathrm{H}-\mathrm{NMR} \quad(500 \mathrm{MHz}$, DMSO- $d_{6}, \delta$ ppm): $10.25(1 \mathrm{H}, \mathrm{s},-\mathrm{CS}-\mathrm{NH}-\mathrm{N}) ; 8.24(2 \mathrm{H}, \mathrm{d}, J=9.0 \mathrm{~Hz}, \mathrm{Ar}-\mathrm{H}) ; 8.01(2 \mathrm{H}, \mathrm{d}, \mathrm{J}=9.0$ $\mathrm{Hz}, \mathrm{Ar}-\mathrm{H}) ; 7.72(1 \mathrm{H}, \mathrm{s}, \mathrm{NH}-\mathrm{C}) ; 2.36\left(3 \mathrm{H}, \mathrm{s}, \mathrm{CH}_{3}\right) ; 2.28(6 \mathrm{H}, \mathrm{brs}$, adamantane- $\mathrm{H}) ; 2.08$ (3H, brs, adamantane-H); 1.66 (6H, brs, adamantane-H). ${ }^{13} \mathrm{C}-\mathrm{NMR}\left(125 \mathrm{MHz}, \mathrm{DMSO}-d_{6}, \delta \mathrm{ppm}\right): 175.6(1 \mathrm{C}, \mathrm{CS})$; 147.5 (1C, Ar-C), 144.8 (1C, N=C); 144.0 (1C, Ar-C); 127.3 (2C, Ar-C); 123.6 (2C, Ar-C); 53.3 (C-NH); 40.7 (3C, adamantane-C); 35.9 (3C, adamantane-C); 29.0 (3C, adamantane-C); $14.3\left(1 \mathrm{C}, \mathrm{CH}_{3}\right)$. ESI-MS [m/z]: $[\mathrm{M}+\mathrm{H}]^{+}=372.9 ;[\mathrm{M}-\mathrm{H}]^{-}=370.9$.

4-(N-Adamantan-1-yl)-1-[1-(3-nitro-4-bromo-phenyl)ethylidene]thiosemicarbazone (3f): ${ }^{1} \mathrm{H}-\mathrm{NMR}(500 \mathrm{MHz}$, DMSO- $d_{6}, \delta$ ppm): $10.22(1 \mathrm{H}, \mathrm{s},-\mathrm{CS}-\mathrm{NH}-\mathrm{N}) ; 8.33(1 \mathrm{H}, \mathrm{d}, J=1.5 \mathrm{~Hz}, \mathrm{Ar}-\mathrm{H}) ; 7.96-7.92(2 \mathrm{H}, \mathrm{m}, \mathrm{Ar}-\mathrm{H})$; $7.69(1 \mathrm{H}, \mathrm{s}, \mathrm{NH}-\mathrm{C}) ; 2.33\left(3 \mathrm{H}, \mathrm{s}, \mathrm{CH}_{3}\right) ; 2.28(6 \mathrm{H}, \mathrm{s}$, adamantane-H); 2.08 (3H, brs, adamantane-H); 1.66 (6H, brs, adamantane-H). ${ }^{13} \mathrm{C}-\mathrm{NMR}\left(125 \mathrm{MHz}, \mathrm{DMSO}-d_{6}, \delta \mathrm{ppm}\right): 175.5$ (1C, CS); 149.9 (1C, Ar-C); 143.9 (1C, N=C); 138.7 (1C; Ar-C); 134.6 (1C; Ar-C); 130.7 (1C; Ar-C); 122.7 (1C; Ar-C); 113.1 (1C, Ar-C); 53.2 (C-NH); 40.6 (3C, adamantane-C); 35.8 (3C, adamantane-C); 28.9 (3C, adamantane-C); 14.0 (1C, $\left.\mathrm{CH}_{3}\right)$. ESI-MS $[\mathrm{m} / \mathrm{z}]:[\mathrm{M}+\mathrm{H}]^{+}=452.9 ;[\mathrm{M}-\mathrm{H}]^{-}=450.8$.

4-(N-Adamantan-1-yl)-1-[1-(4-chloro-phenyl)ethylidene]thiosemicarbazone (3g): ${ }^{1} \mathrm{H}-\mathrm{NMR}(500 \mathrm{MHz}$, DMSO- $d_{6}, \delta$ ppm): $10.07(1 \mathrm{H}, \mathrm{s},-\mathrm{CS}-\mathrm{NH}-\mathrm{N}) ; 7.77(2 \mathrm{H}, \mathrm{d}, J=8.5 \mathrm{~Hz}, \mathrm{Ar}-\mathrm{H}) ; 7.67(1 \mathrm{H}, \mathrm{s}, \mathrm{NH}-\mathrm{C})$; $7.47(2 \mathrm{H}, \mathrm{d}, J=8.5 \mathrm{~Hz}, \mathrm{Ar}-\mathrm{H}) ; 2.30\left(3 \mathrm{H}, \mathrm{s}, \mathrm{CH}_{3}\right) ; 2.27(6 \mathrm{H}, \mathrm{s}$, adamantane-H); $2.08(3 \mathrm{H}, \mathrm{s}$, adamantane- $\mathrm{H})$; $1.66\left(6 \mathrm{H}, \mathrm{s}\right.$, adamantane-H). ${ }^{13} \mathrm{C}-\mathrm{NMR}\left(125 \mathrm{MHz}, \mathrm{DMSO}-d_{6}, \delta \mathrm{ppm}\right): 175.5$ (1C, CS); $146.0(1 \mathrm{C}$, $\mathrm{N}=\mathrm{C}) ; 136.5$ (1C, Ar-C); 133.9 (1C, Ar-C); 128.4 (2C, Ar-C); 127.9 (2C, Ar-C); 53.0 (C-NH); 40.8 (3C, adamantane-C); 35.8 (3C, adamantane-C); 28.9 (3C, adamantane-C); $14.1\left(1 \mathrm{C}, \mathrm{CH}_{3}\right)$. ESI-MS [ $\left.\mathrm{m} / \mathrm{z}\right]$ : [M + $\mathrm{H}]^{+}=361.9 ;[\mathrm{M}-\mathrm{H}]^{-}=359.9$.

4-(N-Adamantan-1-yl)-1-[1-(4-methylphenyl)ethylidene]thiosemicarbazone (3h): ${ }^{1} \mathrm{H}-\mathrm{NMR}(\delta \mathrm{ppm}): 9.69(1 \mathrm{H}$, s, -CS-NH-N); 7.64-7.64 (3H, m, Ar-H and NH-C); 7.22 (2H, d, J = 7.0 Hz, Ar-H); 2.33 (3H, s, $\left.\mathrm{CH}_{3}\right) ; 2.29$ $\left(9 \mathrm{H}, \mathrm{s}, \mathrm{CH}_{3}\right.$ and adamantane-H); $2.09(3 \mathrm{H}, \mathrm{s}$, adamantane- $\mathrm{H}) ; 1.68\left(6 \mathrm{H}, \mathrm{s}\right.$, adamantane-H). ${ }^{13} \mathrm{C}-\mathrm{NMR}(\delta$ ppm): 175.6 (1C, CS); 147.1 (1C, N=C); 138.7 (1C, Ar-C); 134.9 (1C, Ar-C); 128.9 (2C, Ar-C); 125.8 (2C, Ar-C); 52.9 (C-NH); 40.9 (3C, adamantane-C); 35.8 (3C, adamantane-C); 28.9 (3C, adamantane-C); 20.5 $\left(1 \mathrm{C}, \mathrm{CH}_{3}\right) ; 13.8\left(1 \mathrm{C}, \mathrm{CH}_{3}\right)$. ESI-MS $[\mathrm{m} / \mathrm{z}]:[\mathrm{M}+\mathrm{H}]^{+}=342.0$.

4-(N-Adamantan-1-yl)-1-[1-(3-nitro-4-methoxyphenyl)ethylidene]thiosemicarbazone (3i). ${ }^{1} \mathrm{H}-\mathrm{NMR}(\delta \mathrm{ppm})$ : $9.83(1 \mathrm{H}, \mathrm{s},-\mathrm{CS}-\mathrm{NH}-\mathrm{N}) ; 8.18(1 \mathrm{H}, \mathrm{d}, J=2.0 \mathrm{~Hz}, \mathrm{Ar}-\mathrm{H}) ; 8.02(1 \mathrm{H}, \mathrm{dd}, J 1=2.0 \mathrm{~Hz}, J 2=8.5 \mathrm{~Hz}, \mathrm{Ar}-\mathrm{H}) ; 7.64$ $(1 \mathrm{H}, \mathrm{s}, \mathrm{NH}-\mathrm{C}) ; 7.40(1 \mathrm{H}, \mathrm{d}, \mathrm{J}=9.0 \mathrm{~Hz}, \mathrm{Ar}-\mathrm{H}) ; 2.32\left(3 \mathrm{H}, \mathrm{s}, \mathrm{CH}_{3}\right) ; 2.29(6 \mathrm{H}, \mathrm{s}$, adamantane-H); $2.10(3 \mathrm{H}, \mathrm{s}$, 
adamantane-H); 1.68 (6H, s, adamantane-H). ${ }^{13} \mathrm{C}-\mathrm{NMR}(\delta \mathrm{ppm}): 175.4$ (1C, CS); 152.3 (1C, Ar-C); 144.9 (1C; N=C); 139.3 (1C; Ar-C); 131.6 (1C, Ar-C); 130.1 (1C, Ar-C); 122.4 (1C, Ar-C); 114.3 (1C, Ar-C); 53.0 (C-NH); 40.7 (3C, adamantane-C); 35.9 (3C, adamantane-C); 28.9 (3C, adamantane-C); $14.0\left(1 \mathrm{C}, \mathrm{CH}_{3}\right)$. ESI-MS [m/z]: $[\mathrm{M}+\mathrm{H}]^{+}=403.0 ;[\mathrm{M}-\mathrm{H}]^{-}=400.9$.

4-(N-Adamantan-1-yl)-1-[1-(3-nitro-4-chlorophenyl)ethylidene]thiosemicarbazone $(3 \mathbf{j}) .{ }^{1} \mathrm{H}-\mathrm{NMR}(\delta \mathrm{ppm})$ : $10.22(1 \mathrm{H}, \mathrm{s},-\mathrm{CS}-\mathrm{NH}-\mathrm{N}) ; 8.38(1 \mathrm{H}, \mathrm{d}, J=1.5 \mathrm{~Hz}, \mathrm{Ar}-\mathrm{H}) ; 8.07-8.05(1 \mathrm{H}, \mathrm{dd}, J 1=1.5 \mathrm{~Hz}, J 2=8.5 \mathrm{~Hz}$, Ar-H); $7.8(1 \mathrm{H}, \mathrm{d}, J=8.5 \mathrm{~Hz}, \mathrm{Ar}-\mathrm{H}) ; 7.69(1 \mathrm{H}, \mathrm{s}, \mathrm{NH}-\mathrm{C}) ; 2.33\left(3 \mathrm{H}, \mathrm{s}, \mathrm{CH}_{3}\right) ; 2.28$ (3H, s, adamantane-H); 2.08 (3H, s, adamantane-H); $1.66\left(6 \mathrm{H}, \mathrm{s}\right.$, adamantane-H). ${ }^{13} \mathrm{C}-\mathrm{NMR}(\delta \mathrm{ppm}): 175.5(1 \mathrm{C}, \mathrm{CS}) ; 147.8(1 \mathrm{C}$, Ar-C); 143.8 (1C, C=N); 138.2 (1C, Ar-C); 131.6 (1C, Ar-C); 130.8 (1C, Ar-C); 124.9 (1C, Ar-C); 122.8 (1C, Ar-C); 53.2 (C-NH); 40.6 (3C, adamantane-C); 35.9 (3C, adamantane-C); 28.9 (3C, adamantane-C); 14.0 $\left(1 \mathrm{C}, \mathrm{CH}_{3}\right)$. ESI-MS $[\mathrm{m} / \mathrm{z}]:[\mathrm{M}+\mathrm{H}]^{+}=406.9 ;[\mathrm{M}-\mathrm{H}]^{-}=404.9$.

Spectra of the synthesized compounds can be found in the Supplemental Material file.

\subsection{Dertemination of Antimicrobial Activity by the Dilution Method}

The synthesized compounds $\mathbf{2} \mathbf{a}-\mathbf{k}, \mathbf{3 a}-\mathbf{j}$ were dissolved in DMSO at the concentration of $100 \mathrm{mM}$, separately, to prepare the stock solutions. A total of $0.4 \mathrm{~mL}$ of the stock solution was withdrawn and 9.6 mL LB media was added and mixed homogenously to obtain a working solution. Then, twofold serial dilution solutions in LB media was prepared at the concentration from $100 \mu \mathrm{M}$ to $0.78 \mu \mathrm{M}$ in 96 well plates, in triplicate. Before being incubating at $37^{\circ} \mathrm{C}$ for $24 \mathrm{~h}, 50 \mu \mathrm{L}$ of test microorganism suspension at $2 \times 10^{5} \mathrm{CFU} / \mathrm{mL}$ was inoculated to each well. Streptomycin and cycloheximide were used as the positive control samples. Minimum inhibitory concentration (MIC) was defined as the lowest concentration that completely inhibited the development of the microorganism, which was detected by the naked eye. Half of the maximum inhibitory concentration $\left(\mathrm{IC}_{50}\right)$ is defined as the concentration of tested compounds that inhibit $50 \%$ visual growth of the test microorganism, which was determined by using turbidity measurement on a iMark ${ }^{\mathrm{TM}}$ Microplate Absorbance Reader (Bio-Rad Laboratories, Hercules, CA, USA) [48].

\subsection{Determination of Cytotoxicity Activity}

The MTT (3-(4,5-dimethythiazol-2-yl)-2,5-diphenyltetrazolium bromide) method was used to determine the cytotoxicity of the synthesized compounds $\mathbf{2} \mathbf{a}-\mathbf{k}, \mathbf{3} \mathbf{a}-\mathbf{j}$ on human cancer cell lines, i.e., Hep3B (hepatic cancer cell line), HeLa (human cervical cancer cell line), A549 (human lung cancer cell line), and MCF-7 (human breast carcinoma), as described in a previous publication [46]. Tested cells were seeded at $5 \times 10^{6}$ cell/well in 96-well plates with RPMI 1640 or DMEM media containing $10 \%$ fetal bovine serum, penicillin $(100 \mathrm{IU} / \mathrm{mL})$, and streptomycin $(100 \mu \mathrm{g} / \mathrm{mL})$ at $37^{\circ} \mathrm{C}$ in a humid air incubator supplied with $5 \% \mathrm{CO}_{2}$. The cells were stabilized for $24 \mathrm{~h}$ and then were removed to old media and treated with the sample. Each $200 \mu \mathrm{L}$ of the test samples was added to the well and incubated during $72 \mathrm{~h}$ in culture conditions. Following removing the medium, $50 \mu \mathrm{L}$ MTT solution ( $1 \mathrm{mg} / \mathrm{mL}$ in phosphate buffer saline) was then poured into to each well and the cells continue to be incubated at $37^{\circ} \mathrm{C}$ for $4 \mathrm{~h}$. After eliminating the MTT solution, $100 \mu \mathrm{L}$ of isopropanol was added to each well. The absorbance was measured on a iMark ${ }^{\mathrm{TM}}$ Microplate Absorbance Reader (Bio-Rad Laboratories, Hercules, CA, USA) at $570 \mathrm{~nm}$. Suitable blank and positive controls (camptothecin) were included. Cytotoxicity of the synthesized compounds was defined as the percent of cell survival, as follows: [OD (72 h)-OD (0 h)]/[OD(DMSO)-OD (0 h)]; where OD (72 h), OD (0 h), OD(DMSO) are the absorbance of the test sample at $72 \mathrm{~h}$, test sample at $0 \mathrm{~h}$, and the DMSO sample.

\section{Conclusions}

The synthesis and characterization of thiosemicarbazones containing adamantane skeletons $\mathbf{2} \mathbf{a}-\mathbf{k}$ and $\mathbf{3} \mathbf{a}-\mathbf{j}$, were achieved. The syntheses were performed by condensing 4-(1-adamantyl)-3-thiosemicarbazide (1) with substituted benzaldehydes to get compounds 2a-k, 
and with substituted acetophenones to get compounds $3 \mathbf{a}-\mathbf{j}$. The antimicrobial and cytotoxicity of the synthesized compounds were determined. The screening results showed that all synthesized thiosemicarbazones have good inhibitory activity against CA. Among them, compounds $\mathbf{2 c}, \mathbf{2 d}, \mathbf{2 g}$, $\mathbf{2} \mathbf{j}$ and $\mathbf{3 a}, \mathbf{3 e}, \mathbf{3 g}$ displayed the inhibitory activity against $\mathrm{EF}$. Compounds $\mathbf{2 a}, \mathbf{2 e}, \mathbf{2} \mathbf{h}, \mathbf{2} \mathbf{k}$ and $\mathbf{3} \mathbf{j}$ were moderate inhibitors against SA. Compounds $2 \mathrm{a}, \mathbf{2 e}$ and $\mathbf{2 g}$ were found to have so good inhibitory effect on BC. Screening of structure-antimicrobial activity relationship of thiosemicarbazones which were synthesized by condensing 4-(1-adamantyl)-3-thiosemicarbazide (1) with substituted benzaldehydes, replacing the $-\mathrm{H}$ atom by substituents on the phenyl ring improved the inhibition against $\mathrm{EF}$ and $\mathrm{CA}$ but decreased that of SA and BC. Nevertheless, in case the compounds were formed by condensing 4-(1-adamantyl)-3-thiosemicarbazide (1) with substituted acetophenones, the inhibition against EF, $\mathrm{SA}, \mathrm{BC}$ and CA seemed to decrease if replacing $-\mathrm{H}$ atom by substituents on the phenyl ring (except compounds $\mathbf{3 e}, \mathbf{3 g}$ and $\mathbf{3 f}$ ). Among all synthesized thiosemicarbazones, compounds $\mathbf{2} \mathbf{d}$ and $\mathbf{2 h}$, which contained (ortho) hydroxyl groups on the phenyl ring, showed good inhibitory activity against the tested cancer cell lines, i.e., Hep3B, A549, and MCF-7. Moreover, compounds $\mathbf{2 a - c , ~} \mathbf{2 f}, \mathbf{2 g}, \mathbf{2 j}, \mathbf{2} \mathbf{2 k}, \mathbf{3 g}$, and $3 \mathbf{i}$ were moderate inhibitors against MCF-7.

Supplementary Materials: Supplementary materials can be obtained online.

Author Contributions: The manuscript was written by the contribution of all authors. Methodology: D.C.P. and V.H.P.; supervision: B.D.V.; writing—original draft: V.H.P.; writing—review and editing: T.P.D.P. All authors have read and agreed to the published version of the manuscript.

Funding: This research received no external funding.

Conflicts of Interest: The authors declare there are no conflict of interests.

\section{References}

1. Haribabu, J.; Subhashree, G.R.; Saranya, S.; Gomathi, K.; Karvembu, R.; Gayathri, D. Isatin based thiosemicarbazone derivatives as potential bioactive agents: Anti-oxidant and molecular docking studies. J. Mol. Struct. 2016, 1110, 185-195. [CrossRef]

2. Ghosh, S.; Misra, A.K.; Bhatia, G.; Khan, M.M.; Khanna, A.K. Syntheses and evaluation of glucosyl aryl thiosemicarbazide and glucosyl thiosemicarbazone derivatives as antioxidant and anti-dyslipidemic agents. Bioorg. Med. Chem. Lett. 2009, 19, 386-389. [CrossRef]

3. Tenorio, R.P.; Carvalho, C.S.; Pessanha, C.S.; de Lima, J.G.; de Faria, A.R.; Alves, A.J.; de Melo, E.J.T.; Goes, A.J.S. Synthesis of thiosemicarbazone and 4-thiazolidinone derivatives and their in vitro anti-Toxoplasma gondii activity. Bioorg. Med. Chem. Lett. 2005, 15, 2575-2578. [CrossRef]

4. Bharti, N.; Husain, K.; Garza, M.T.G.; Cruz-Vega, D.E.; Castro-Garza, J.; Mata-Cardenas, B.D.; Naqvi, F.; Azam, A. Synthesis and in vitro antiprotozoal activity of 5-nitrothiophene-2-carboxaldehyde thiosemicarbazone derivatives. Bioorg. Med. Chem. Lett. 2002, 12, 3475-3478. [CrossRef]

5. de Oliveira, R.B.; de Souza-Fagundes, E.M.; Soares, R.P.P.; Andrade, A.A.; Krettli, A.U.; Zani, C.L. Synthesis and antimalarial activity of semicarbazone and thiosemicarbazone derivatives. Eur. J. Med. Chem. 2008, 43, 1983-1988. [CrossRef] [PubMed]

6. Shailendra, N.S.; Bharti, N.; Garza, M.T.G.; Cruz-Vega, D.E.; Garza, J.C.; Saleem, K.; Naqvi, F.; Azam, A. Synthesis, characterisation and antiamoebic activity of new thiophene-2-carboxaldehyde thiosemicarbazone derivatives and their cyclooctadiene Ru (II) complexes. Bioorg. Med. Chem. Lett. 2001, 11, 2675-2678. [CrossRef]

7. Dimmock, J.R.; McColl, J.M.; Wonko, S.L.; Thayer, R.S.; Hancock, D.S. Evaluation of the thiosemicarbazones of some aryl alkyl ketones and related compounds for anticonvulsant activities. Eur. J. Med. Chem. 1991, 26, 529-534. [CrossRef]

8. Bal, T.R.; Anand, B.; Yogeeswari, P.; Sriram, D. Synthesis and evaluation of anti-HIV activity of isatin $\beta$-thiosemicarbazone derivatives. Bioorg. Med. Chem. Lett. 2005, 15, 4451-4455. [CrossRef] [PubMed] 
9. Finkielsztein, L.M.; Castro, E.F.; Fabián, L.E.; Moltrasio, G.Y.; Campos, R.H.; Cavallaro, L.V.; Moglioni, A.G. New 1-indanone thiosemicarbazone derivatives active against BVDV. Eur. J. Med. Chem. 2008, 43, 1767-1773. [CrossRef] [PubMed]

10. Shipman, J.C.; Smith, S.H.; Drach, J.C.; Klayman, D.L. Antiviral activity of 2-acetylpyridine thiosemicarbazones against herpes simplex virus. Antimicrob. Agents Chemother. 1981, 19, 682. [CrossRef]

11. Khan, S.A.; Kumar, P.; Joshi, R.; Iqbal, P.F.; Saleem, K. Synthesis and in vitro antibacterial activity of new steroidal thiosemicarbazone derivatives. Eur. J. Med. Chem. 2008, 43, 2029-2034. [CrossRef] [PubMed]

12. Kulandaivelu, U.; Padmini, V.G.; Suneetha, K.; Shireesha, B.; Vidyasagar, J.V.; Rao, T.R.; Basu, A.; Jayaprakash, V. Synthesis, antimicrobial and anticancer activity of new thiosemicarbazone derivatives. Arch. Pharm. 2011, 344, 84-90. [CrossRef]

13. Sriram, D.; Yogeeswari, P.; Thirumurugan, R.; Pavana, R.K. Discovery of new antitubercular oxazolyl thiosemicarbazones. J. Med. Chem. 2006, 49, 3448-3450. [CrossRef] [PubMed]

14. Altıntop, M.D.; Atlı, Ö.; Ilgın, S.; Demirel, R.; Özdemir, A.; Kaplancıklı, Z.A. Synthesis and biological evaluation of new naphthalene substituted thiosemicarbazone derivatives as potent antifungal and anticancer agents. Eur. J. Med. Chem. 2016, 108, 406-414. [CrossRef] [PubMed]

15. Degola, F.; Morcia, C.; Bisceglie, F.; Mussi, F.; Tumino, G.; Ghizzoni, R.; Pelosi, G.; Terzi, V.; Buschini, A.; Restivo, F.M.; et al. In vitro evaluation of the activity of thiosemicarbazone derivatives against mycotoxigenic fungi affecting cereals. Int. J. Food. Microbiol. 2015, 200, 104-111. [CrossRef] [PubMed]

16. Piantanida, I.; Cindric, M. Novel thiosemicarbazone derivatives as potential antitumor agents: synthesis, physicochemical and structural properties, DNA interactions and antiproliferative activity. Bioorg. Med. Chem. 2008, 16, 5189-5198.

17. Feun, L.; Modiano, M.; Lee, K.; Mao, J.; Marini, A.; Savaraj, N.; Plezia, P.; Almassian, B.; Colacino, E.; Fischer, J. Phase I and pharmacokinetic study of 3-aminopyridine-2-carboxaldehyde thiosemicarbazone (3-AP) using a single intravenous dose schedule. Cancer Chemother. Pharmacol. 2002, 50, 223-229. [CrossRef]

18. Hu, K.; Yang, Z.H.; Pan, S.S.; Xu, H.J.; Ren, J. Synthesis and antitumor activity of liquiritigenin thiosemicarbazone derivatives. Eur J. Med. Chem. 2010, 45, 3453-3458. [CrossRef]

19. Wang, Y.Y.; Gu, W.; Shan, Y.; Liu, F.; Xu, X.; Yang, Y.Q.; Zhang, Q.J.; Zhang, Y.; Kuang, H.B.; Wang, Z.L. Design, synthesis and anticancer activity of novel nopinone-based thiosemicarbazone derivatives. Bioorg. Med. Chem. Lett. 2017, 27, 2360-2363. [CrossRef]

20. de Oliveira, J.F.; Lima, T.S.; Vendramini-Costa, D.B.; de Lacerda Pedrosa, S.C.B.; Lafayette, E.A.; da Silva, R.M.F.; de Almeida, S.M.V.; de Moura, R.O.; Ruiz, A.L.T.G.; de Carvalho, J.E. Thiosemicarbazones and 4-thiazolidinones indole-based derivatives: Synthesis, evaluation of antiproliferative activity, cell death mechanisms and topoisomerase inhibition assay. Eur. J. Med. Chem. 2017, 136, 305-314. [CrossRef]

21. Davies, W.L.; Grunert, R.R.; Haff, R.F.; McGahen, J.W.; Neumayer, E.M.; Paulshock, M.; Watts, J.C.; Wood, T.R.; Hermann, E.C.; Hoffmann, C.E. Antiviral activity of 1-adamantanamine (amantadine). Science 1964, 144, 862-863. [CrossRef]

22. Wendel, H.A.; Snyder, M.T.; Pell, S. Trial of amantadine in epidemic influenza. Clin. Pharmacol. Ther. 1966, 7, 38-43. [CrossRef] [PubMed]

23. Vernier, V.G.; Harmon, J.B.; Stump, J.M.; Lynes, T.E.; Marvel, J.P.; Smith, D.H. The toxicologic and pharmacologic properties of amantadine hydrochloride. Toxicol. Appl. Pharmacol. 1969, 15, 642-665. [CrossRef]

24. Tilley, J.W.; Levitan, P.; Kramer, M.J. Adamantylthiourea derivatives as antiviral agents. J. Med. Chem. 1979, 22, 1009-1010. [CrossRef] [PubMed]

25. Aigami, K.; Inamoto, Y.; Takaishi, N.; Hattori, K.; Takatsuki, A.; Tamura, G. Biologically active polycycloalkanes. 1. Antiviral adamantane derivatives. J. Med. Chem. 1975, 18, 713-721. [CrossRef] [PubMed]

26. Basarić, N.; Sohora, M.; Cindro, N.; Mlinarić-Majerski, K.; De Clercq, E.; Balzarini, J. Antiproliferative and antiviral activity of three libraries of adamantane derivatives. Arch. Pharm. 2014, 347, 334-340. [CrossRef] [PubMed] 
27. Hassan, G.S.; El-Emam, A.A.; Gad, L.M.; Barghash, A.E.M. Synthesis, antimicrobial and antiviral testing of some new 1-adamantyl analogues. Saudi Pharm. J. 2010, 18, 123-128. [CrossRef]

28. Göktaş, F.; Vanderlinden, E.; Naesens, L.; Cesur, N.; Cesur, Z. Microwave assisted synthesis and anti-influenza virus activity of 1-adamantyl substituted N-(1-thia-4-azaspiro[4.5]decan-4-yl)carboxamide derivatives. Bioorg. Med. Chem. 2012, 20, 7155-7159. [CrossRef]

29. El-Emam, A.A.; Al-Deeb, O.A.; Al-Omar, M.; Lehmann, J. Synthesis, antimicrobial, and anti-HIV-1 activity of certain 5-(1-adamantyl)-2-substituted thio-1,3,4-oxadiazoles and 5-(1-adamantyl)-3-substituted aminomethyl-1,3,4-oxadiazoline-2-thiones. Bioorg. Med. Chem. 2004, 12, 5107-5113. [CrossRef]

30. Orzeszko, A.; Kamińska, B.; Orzeszko, G.; Starościak, B.J. Synthesis and antimicrobial activity of new adamantane derivatives II. Il Farm. 2000, 55, 619-623. [CrossRef]

31. Orzeszko, A.; Gralewska, R.; Starościak, B.J.; Kazimierczuk, Z. Synthesis and antimicrobial activity of new adamantane derivatives I. Acta Biochim. Pol. 2000, 47, 87-94. [CrossRef] [PubMed]

32. Wang, J.J.; Wang, S.S.; Leeb, C.F.; Chung, M.A.; Chern, Y.T. In vitro antitumor and antimicrobial activities of $\mathrm{N}$-substituents of maleimide by adamantane and diamantane. Chemotherapy 1997, 43, 182-189. [CrossRef] [PubMed]

33. Al-Wahaibi, L.; Hassan, H.; Abo-Kamar, A.; Ghabbour, H.; El-Emam, A. Adamantane-isothiourea hybrid derivatives: Synthesis, characterization, in vitro antimicrobial, and in vivo hypoglycemic activities. Molecules 2017, 22, 710. [CrossRef] [PubMed]

34. Balaji, G.L.; Sarveswari, S.; Vijayakumar, V. Synthesis of diversely substituted adamantanes as a new class of antimicrobial agent. Res. Chem. Intermed. 2015, 41, 6765-6776. [CrossRef]

35. Al-Abdullah, E.; Al-Tuwaijri, H.; Hassan, H.; Al-Alshaikh, M.; Habib, E.; El-Emam, A. Synthesis, antimicrobial and hypoglycemic activities of novel N-(1-adamantyl) carbothioamide derivatives. Molecules 2015, 20, 8125-8143. [CrossRef] [PubMed]

36. Tabbi, A.; Tebbani, D.; Caporale, A.; Saturnino, C.; Nabavi, S.F.; Giuseppe, P.; Arra, C.; Canturk, Z.; Turan-Zitouni, G.; Merazig, H. New Adamantyl Chalcones: Synthesis, Antimicrobial and Anticancer Activities. Curr. Top. Med. Chem. 2017, 17, 498-506. [CrossRef]

37. Fesatidou, M.; Zagaliotis, P.; Camoutsis, C.; Petrou, A.; Eleftheriou, P.; Tratrat, C.; Haroun, M.; Geronikaki, A.; Ciric, A.; Sokovic, M. 5-Adamantan thiadiazole-based thiazolidinones as antimicrobial agents. Design, synthesis, molecular docking and evaluation. Bioorg. Med. Chem. 2018, 26, 4664-4676. [CrossRef]

38. El-Emam, A.A.; Al-Tamimi, A.M.S.; Al-Omar, M.A.; Alrashood, K.A.; Habib, E.E. Synthesis and antimicrobial activity of novel 5-(1-adamantyl)-2-aminomethyl-4-substituted-1,2,4-triazoline-3-thiones. Eur. J. Med. Chem. 2013, 68, 96-102. [CrossRef]

39. El-Emam, A.A.; Alrashood, K.A.; Al-Omar, M.A.; Al-Tamimi, A.M.S. Synthesis and antimicrobial activity of $\mathrm{N}^{\prime}$-heteroarylidene-1-adamantylcarbohydrazides and (+/-)-2-(1-adamantyl)-4-acetyl-5-[5-(4-substituted phenyl-3-isoxazolyl)]-1,3,4-oxadiazolines. Molecules 2012, 17, 3475-3483. [CrossRef]

40. Aguiar, D.F.; Dutra, L.L.A.; Dantas, W.M.; Camelo de Carvalho, G.G.; Gonçalves Lemes, R.P.; do Ó Pessoa, C.; Koscky Paier, C.R.; Barros Araujo, P.L.; Araujo, E.S.; Pena, L.J. Synthesis, Antitumor and Cytotoxic Activity of New Adamantyl O-Acylamidoximes and 3-Aryl-5-Adamantane-1, 2, 4-Oxadiazole Derivatives. Chem. Sel. 2019, 4, 9112-9118. [CrossRef]

41. Anusha, S.; Mohan, C.D.; Ananda, H.; Baburajeev, C.P.; Rangappa, S.; Mathai, J.; Fuchs, J.E.; Li, F.; Shanmugam, M.K.; Bender, A.; et al. Adamantyl-tethered-biphenylic compounds induce apoptosis in cancer cells by targeting Bcl homologs. Bioorg. Med. Chem. Lett. 2016, 26, 1056-1060. [CrossRef] [PubMed]

42. Fytas, C.; Zoidis, G.; Tsotinis, A.; Fytas, G.; Khan, M.A.; Akhtar, S.; Rahman, K.M.; Thurston, D.E. Novel 1-(2-aryl-2-adamantyl)piperazine derivatives with antiproliferative activity. Eur. J. Med. Chem. 2015, 93, 281-290. [CrossRef]

43. Cincinelli, R.; Musso, L.; Giannini, G.; Zuco, V.; De Cesare, M.; Zunino, F.; Dallavalle, S. Influence of the adamantyl moiety on the activity of biphenylacrylohydroxamic acid-based HDAC inhibitors. Eur. J. Med. Chem. 2014, 79, 251-259. [CrossRef] [PubMed]

44. Zhu, X.H.; Sun, J.; Wang, S.S.; Bu, W.; Yao, M.N.; Gao, K.; Song, Y.; Zhao, J.Y.; Lu, C.T.; Zhang, E.H.; et al. Synthesis, crystal structure, superoxide scavenging activity, anticancer and docking studies of novel adamantyl nitroxide derivatives. J. Mol. Struct. 2016, 1108, 611-617. [CrossRef]

45. Pham, V.H.; Phan, T.P.D.; Phan, D.C.; Vu, B.D. Synthesis and Bioactivity of Hydrazide-Hydrazones with the 1-Adamantyl-Carbonyl Moiety. Molecules 2019, 24, 4000. [CrossRef] 
46. Mosmann, T. Rapid colorimetric assay for cellular growth and survival: application to proliferation and cytotoxicity assays. J. Immunol. Methods 1983, 65, 55-63. [CrossRef]

47. Reis, C.M.; Pereira, D.S.; Paiva, R.O.; Kneipp, L.F.; Echevarria, A. Microwave-assisted synthesis of new N1, N4-substituted thiosemicarbazones. Molecules 2011, 16, 10668-10684. [CrossRef]

48. Wiegand, I.; Hilpert, K.; Hancock, R.E.W. Agar and broth dilution methods to determine the minimal inhibitory concentration (MIC) of antimicrobial substances. Nat. Protoc. 2008, 3, 163. [CrossRef]

Sample Availability: Samples of the compound are available from the authors.

(C) 2020 by the authors. Licensee MDPI, Basel, Switzerland. This article is an open access article distributed under the terms and conditions of the Creative Commons Attribution (CC BY) license (http://creativecommons.org/licenses/by/4.0/). 